\title{
A Concise and Atom-Economical Suzuki-Miyaura Coupling Reaction Using Unactivated Trialkyl- and Triarylboranes with Aryl Halides
}

Hongmei Li*, Yong-Li Zhong*, Cheng-yi Chen, Ashley E. Ferraro and Dengjin Wang

Process Chemistry, Merck Research Laboratories, P.O. Box 2000, Rahway, NJ 07065, USA

hongmei06@gmail.com \& yongli_zhong@merck.com

\section{Table of Contents}

$\begin{array}{lll}\text { Part I. General Information } & \text { S2 }\end{array}$

Part II. $\quad$ Experimental Procedures and NMR data S2-S8

Part III. $\quad{ }^{1} \mathrm{H}$ and ${ }^{13} \mathrm{C}$ NMR Spectra $\quad$ S9-S40

$\begin{array}{lll}\text { Part IV. } & \text { References } & \text { S41 }\end{array}$ 
General Information. Commercial reagents and anhydrous solvents were used without further purification. All reactions were conducted under $\mathrm{N}_{2}$ atmosphere, and monitored by High Performance Liquid Chromatography (HPLC) (using Ascentis Express C18 $2.7 \mu \mathrm{m}, 100 \mathrm{~mm} \times 4.6 \mathrm{~mm}$ ) or GC. Concentration in vacuum refers to removal of the solvent using a rotary evaporator at reduced pressure (10-20 torr) with water bath. ${ }^{1} \mathrm{H}$ NMR and ${ }^{13} \mathrm{C}$ NMR spectra were recorded on a Bruker Avance 400 or $500 \mathrm{NMR}$ Spectrometer with chemical shifts reported in $\mathrm{ppm}$ relative to the residual $\mathrm{CDCl}_{3}$. High resolving power accurate mass measurements were acquired by use of a Bruker Daltonics 7T Fourier transform ion cyclotron resonance (FTICR) mass spectrometer.

\section{General Suzuki-Miyaura cross-coupling procedure with commercially available trialkyl or triarylboranes (A):}

To a flask charged aryl bromide ( 1 equiv), trialkylborane in THF ( $1 \mathrm{M}, 0.40$ equiv) or triphenylborane (0.40 equiv), $\mathrm{Pd}_{2} \mathrm{dba}_{3} \bullet \mathrm{DCM}$ ( 0.0125 equiv), $n \mathrm{BuAd}_{2} \mathrm{P}$ (0.05 equiv), $\mathrm{K}_{3} \mathrm{PO}_{4}$ ( 2 equiv) and toluene (10 volume) and degassed water (1 volume) under $\mathrm{N}_{2}$, the reaction mixture were heated to $100{ }^{\circ} \mathrm{C}$ under $\mathrm{N}_{2}$ and stirred until HPLC shows full consumption of aryl bromide. The reaction mixture was cooled to $\mathrm{rt}$ and added water $(5 \mathrm{~mL})$ and extracted with EtOAc $(10 \mathrm{~mL})$, cut the layer and back extract the aqueous layer once with EtOAc $(10 \mathrm{~mL})$. The combined organic layers were dried, then assay by HPLC with desired product standard for yield or concentrated down and purified by flash chromatography.

Compound $\mathbf{3 a - 3 i}, \mathbf{4} \mathbf{b}$ and $\mathbf{4} \mathbf{I}$ were prepared based on above procedure $\mathrm{A}$ with commercially available $\mathrm{Et}_{3} \mathrm{~B}, n-\mathrm{Bu}_{3} \mathrm{~B}$, sec $-\mathrm{Bu}_{3} \mathrm{~B}$ and $\mathrm{Ph}_{3} \mathrm{~B}$. All spectral data of isolated coupling products matched that of the commercial reagent.

General Suzuki-Miyaura cross-coupling procedure with trialkylboranes generated in situ via hydroboration of terminal alkenes (B):

To a flask charged $\mathrm{BH}_{3} \bullet \mathrm{THF}\left(1 \mathrm{M}, 0.45\right.$ equiv) under $\mathrm{N}_{2}$, cooled to $0{ }^{\circ} \mathrm{C}$ and added terminal alkene (1.2 equiv) dropwise, aged the mixture at $\mathrm{rt}$ for $1 \mathrm{~h}$. Then degassed water $(0.3 \mathrm{~mL})$ was added to the flask to quench the excess amount of $\mathrm{BH}_{3}$ in the reaction mixture. $\mathrm{K}_{3} \mathrm{PO}_{4}(2$ equiv), toluene $(3 \mathrm{~mL})$, aryl halide ( $0.30 \mathrm{~g}, 1$ equiv), $\mathrm{Pd}_{2} \mathrm{dba}_{3} \bullet \mathrm{DCM}$ (0.0125 equiv) and Ruphos ( 0.05 equiv) were added under $\mathrm{N}_{2}$, the reaction mixture were heated up to $100{ }^{\circ} \mathrm{C}$ and aged under $\mathrm{N}_{2}$ for $\sim 20 \mathrm{~h}$. The reaction mixture was cooled down to rt and added water $(10 \mathrm{~mL})$ and extracted with EtOAc $(20 \mathrm{~mL})$. The aqueous phase was extracted again with EtOAc $(10 \mathrm{~mL})$. The combined organic layers were dried, concentrated and purified by flash chromatography.

\section{General procedure for the kinetics studies (the experiments were set up and run in glove box):}

To a $40 \mathrm{~mL}$ vial was charged bromobenzene ( $8.0 \mathrm{mmol}, 1$ equiv), $\mathrm{K}_{3} \mathrm{PO}_{4}(16.0 \mathrm{mmol}, 2$ equiv), $1 \mathrm{M}$ tri- $n$ butylborane in THF solution ( $2.8 \mathrm{mmol}, 0.35$ equiv; or $5.6 \mathrm{mmol}, 0.70$ equiv) or $n$-butylboronic acid ( 8.4 mmol, 1.05 equiv), $\mathrm{Pd}(\mathrm{OAc})_{2}$ ( $0.20 \mathrm{mmol}, 0.025$ equiv), $n-\mathrm{BuAd}_{2} \mathrm{P}(0.40 \mathrm{mmol}, 0.05$ equiv), degassed water $(1.6 \mathrm{~mL})$. Then, toluene was charged to fill up the reaction mixture to a total of $16 \mathrm{~mL}$, which was sealed with a rubber cap. The reaction mixture were stirred and heated to $90{ }^{\circ} \mathrm{C} .50 \mu \mathrm{L}$ of solution was 
taken from the reaction mixture for every $15 \mathrm{~min}$ or every $30 \mathrm{~min}$. The solution was diluted with $5 \mathrm{~mL}$ of acetonitrile for HPLC.

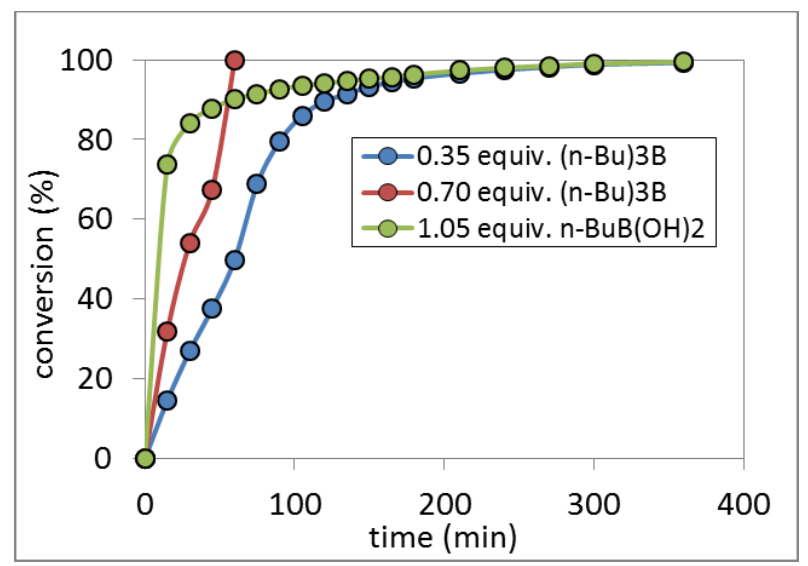

Figure 1. Plot of conversion versus time for 0.35 equiv or 0.70 equiv of tri- $n$-butylborane or 1.05 equiv of $n$-butylboranic acid with 1.0 equiv of bromobenzene $(0.5 \mathrm{M}), 2.5 \mathrm{~mol} \%$ of $\mathrm{Pd}(\mathrm{OAc})_{2}$ and $5 \mathrm{~mol} \%$ of $n-\mathrm{BuAd}_{2} \mathrm{P}$ in $10: 1$ of toluene/water in the Suzuki-Miyaura reaction at $90{ }^{\circ} \mathrm{C}$. The reaction was run in glove box instead of in a fume hood for a typical reaction.<smiles>c1ccc(CCCc2ccccc2)cc1</smiles>

Preparation of 1,3-diphenylpropane (5a):

5a was prepared based on above procedure B, purified by flash chromatography (eluting solvent: hexanes) to give title compound $(\mathrm{X}=\mathrm{Br}, 0.32 \mathrm{~g}$, yield $=86 \%$; and $\mathrm{X}=\mathrm{Cl}, 0.40 \mathrm{~g}$, yield $=76 \%)$ as a colorless oil. ${ }^{\text {i }}{ }^{1} \mathrm{H}$ NMR (400 MHz, $\mathrm{CDCl}_{3}$ ) $\delta$ : 7.32-7.26 (m, $\left.4 \mathrm{H}\right), 7.21-7.17$ (m, $\left.6 \mathrm{H}\right), 2.67$ (t, J = $7.7 \mathrm{~Hz}, 4 \mathrm{H}), 1.98$ (m, $2 \mathrm{H})$.<smiles>c1ccc(CCCOC2CCCCO2)cc1</smiles>

Preparation of 2-(3-phenylpropoxy)tetrahydro-2H-pyran (5b):

5a was prepared based on above procedure $\mathrm{B}$, purified by flash chromatography (eluting solvents: MTBE/hexanes $=0-20 \%)$ to give title compound $\left(0.41 \mathrm{~g}\right.$, yield $=98 \%$ ) as a colorless oil. ${ }^{\text {ii }}{ }^{1} \mathrm{H}$ NMR (400

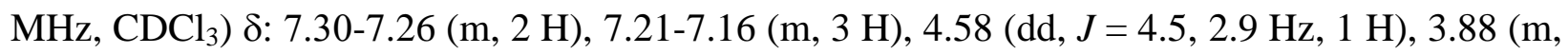
$1 \mathrm{H}), 3.78(\mathrm{~m}, 1 \mathrm{H}), 3.50(\mathrm{~m}, 1 \mathrm{H}), 3.41(1 \mathrm{H}), 2.71(\mathrm{~m}, 2 \mathrm{H}), 1.93(\mathrm{~m}, 2 \mathrm{H}), 1.85(\mathrm{~m}, 1 \mathrm{H}), 1.72$ (m, $1 \mathrm{H}), 1.64-1.50(\mathrm{~m}, 4 \mathrm{H})$. 


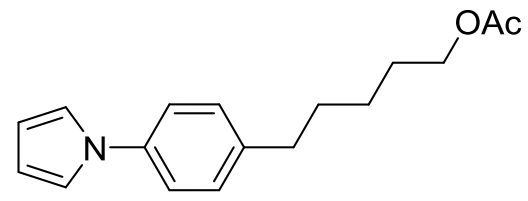

Preparation of 5-(4-(1H-pyrrol-1-yl)phenyl)pentyl acetate (5c):

5c was prepared based on above procedure B, purified by flash chromatography (eluting solvents: EtOAc/hexanes $=0-30 \%)$ to give title compound $(0.28 \mathrm{~g}$, yield $=76 \%)$ as a colorless oil. ${ }^{1} \mathrm{H} \mathrm{NMR}(500$ $\left.\mathrm{MHz} \mathrm{CDCl}_{3}\right) \delta: 1.45(\mathrm{~m}, 2 \mathrm{H}), 1.72(\mathrm{~m}, 4 \mathrm{H}), 2.10(\mathrm{~s}, 3 \mathrm{H}), 2.67(\mathrm{t}, J=6.8 \mathrm{~Hz}, 2 \mathrm{H}), 4.10(\mathrm{t}, J=$ $6.6 \mathrm{~Hz}, 2 \mathrm{H}), 6.39$ (br s, $2 \mathrm{H}), 7.10$ (br s, $2 \mathrm{~h}), 7.25$ (m, $2 \mathrm{H}), 7.34(\mathrm{~m}, 2 \mathrm{H}) ;{ }^{13} \mathrm{C} \mathrm{NMR}(125 \mathrm{MHz}$,

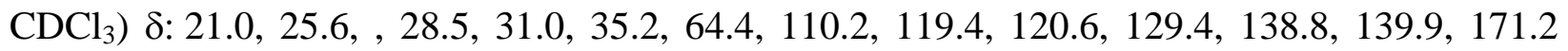
ppm. HRMS (ESI) calculated for $\mathrm{C}_{17} \mathrm{H}_{21} \mathrm{NO}_{2}(\mathrm{M}+\mathrm{H})^{+} 272.1657$, found 272.1645.

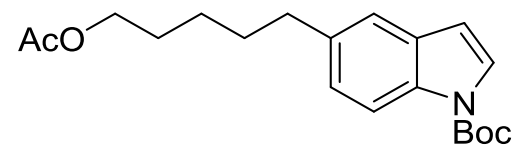

tert-Butyl 5-(5-acetoxypentyl)-1H-indole-1-carboxylate (5d):

5d was prepared based on above procedure B, purified by flash chromatography (eluting solvents: EtOAc/hexanes $=0-30 \%)$ to give title compound $(0.33 \mathrm{~g}$, yield $=94 \%)$ as a colorless oil. ${ }^{1} \mathrm{H}$ NMR $(500$ $\mathrm{MHz}_{\mathrm{CDCl}}$ ) $\delta: 1.41(\mathrm{p}, J=7.6 \mathrm{~Hz}, 2 \mathrm{H}), 1.68(\mathrm{~m}, 13 \mathrm{H}), 2.05(\mathrm{~s}, 3 \mathrm{H}), 2.72(\mathrm{t}, J=7.6 \mathrm{~Hz}, 2 \mathrm{H})$, $4.07(\mathrm{t}, J=6.7 \mathrm{~Hz}, 2 \mathrm{H}), 6.52(\mathrm{~d}, J=3.7 \mathrm{~Hz}, 1 \mathrm{H}), 7.15(\mathrm{~d}, J=8.5 \mathrm{~Hz}, 1 \mathrm{H}), 7.36(\mathrm{~s}, 1 \mathrm{H}), 7.58$ $(\mathrm{s}, 1 \mathrm{H}), 8.06(\mathrm{~s}, 1 \mathrm{H}) ;{ }^{13} \mathrm{C}$ NMR $\left(125 \mathrm{MHz}, \mathrm{CDCl}_{3}\right) \delta: 21.0,25.5,28.2,28.5,31.5,35.6,64.5$, 83.5, 107.1, 114.9, 120.3, 124.9, 126.0, 130.8, 133.6, 136.8, 149.8, 171.2 ppm. HRMS (ESI) calculated for $\mathrm{C}_{20} \mathrm{H}_{27} \mathrm{NO}_{4}(\mathrm{M}+\mathrm{Na})^{+} 368.1851$, found 368.1832 .<smiles>CCOC(CCCc1ccccc1OC)OCC</smiles>

1-(4,4-Diethoxybutyl)-2-methoxybenzene (5e):

5e was prepared based on above procedure $B$, purified by flash chromatography (eluting solvents: EtOAc/hexanes $=0-30 \%)$ to give title compound $(0.40 \mathrm{~g}$, yield $=98 \%)$ as a colorless oil. ${ }^{1} \mathrm{H}$ NMR $(500$ $\mathrm{MHz}_{\mathrm{CDCl}}$ ) $\delta: 1.22(\mathrm{t}, J=7.1 \mathrm{~Hz}, 6 \mathrm{H}), 1.69-1.68(\mathrm{~m}, 4 \mathrm{H}), 2.66(\mathrm{~s}, 2 \mathrm{H}), 3.53-3.47(\mathrm{~m}, 2 \mathrm{H})$, 3.68-3.62 (m, $2 \mathrm{H}), 3.82(\mathrm{~s}, 3 \mathrm{H}), 4.53(\mathrm{~m}, 1 \mathrm{H}), 6.90-6.83(\mathrm{~m}, 2 \mathrm{H}), 7.20-7.14(\mathrm{~m}, 2 \mathrm{H}) ;{ }^{13} \mathrm{C}$ NMR $\left(125 \mathrm{MHz}, \mathrm{CDCl}_{3}\right) \delta: 15.4,25.0,29.9,33.4,55.2,60.9,103.0,110.2,120.4,126.9,129.8$, 130.7, 157.5 ppm. HRMS (ESI) calculated for $\mathrm{C}_{15} \mathrm{H}_{24} \mathrm{O}_{3}(\mathrm{M}+\mathrm{Na})^{+} 275.1626$, found 275.1618. 
<smiles>CCOC(=O)C(C)CCCc1ccc(OC)cc1</smiles>

Ethyl 5-(4-methoxyphenyl)-2-methylpentanoate (5f):

5f was prepared based on above procedure B, purified by flash chromatography (eluting solvents: EtOAc/hexanes $=0-30 \%)$ to give title compound $\left(0.35 \mathrm{~g}\right.$, yield $=88 \%$ ) as a colorless oil. ${ }^{1} \mathrm{H}$ NMR (400 $\left.\mathrm{MHz} \mathrm{CDCl}_{3}\right) \delta: 1.14(\mathrm{~d}, J=7.0 \mathrm{~Hz}, 3 \mathrm{H}), 1.24(\mathrm{t}, J=7.1 \mathrm{~Hz}, 3 \mathrm{H}), 1.48-1.40(\mathrm{~m}, 1 \mathrm{H}), 1.73-$ $1.52(\mathrm{~m}, 3 \mathrm{H}), 2.45-2.40(\mathrm{~m}, 1 \mathrm{H}), 2.55$ (t, $J=7.6 \mathrm{~Hz}, 2 \mathrm{H}), 3.78(\mathrm{~s}, 3 \mathrm{H}), 4.12$ (q, J = 7.1 Hz, 2 $\mathrm{H}), 6.85-6.81(\mathrm{~m}, 2 \mathrm{H}), 7.08(\mathrm{~d}, J=8.3 \mathrm{~Hz}, 2 \mathrm{H}) ;{ }^{13} \mathrm{C} \mathrm{NMR}\left(100 \mathrm{MHz}, \mathrm{CDCl}_{3}\right) \delta: 14.3,17.1$, 29.3, 33.4, 34.8, 39.5, 55.2, 60.1, 113.7, 129.2, 134.3, 157.8, 176.8 ppm. HRMS (ESI) calculated for $\mathrm{C}_{15} \mathrm{H}_{22} \mathrm{O}_{3}(\mathrm{M})^{+} 250.1585$, found 250.1587 .<smiles>FC(F)(F)c1ccc(CCCC[C@@H]2C[C@H]2Sc2ccccc2)cc1</smiles>

Dimethyl(phenyl)((1R,2R)-2-(4-(4-(trifluoromethyl)phenyl)butyl)cyclopropyl)silane (5g):

$\mathbf{5 g}$ was prepared based on above procedure $\mathrm{B}$, purified by flash chromatography (eluting solvents: EtOAc/hexanes $=0-10 \%)$ to give title compound $(\mathrm{X}=\mathrm{Br}, 0.46 \mathrm{~g}$, yield $=92 \%$; and $\mathrm{X}=\mathrm{Cl}, 0.54 \mathrm{~g}$, yield = $87 \%)$ as a colorless oil. ${ }^{1} \mathrm{H} \mathrm{NMR}\left(400 \mathrm{MHz} \mathrm{CDCl}_{3}\right) \delta$ : $-0.40(\mathrm{dt}, J=9.8,6.5 \mathrm{~Hz}, 1 \mathrm{H}), 0.24(\mathrm{~s}, 6$ H), 0.54-0.37 (m, 2 H), 0.72-0.69 (m, $1 \mathrm{H}), 0.97-0.91$ (m, $1 \mathrm{H}), 1.33-1.24$ (m, $2 \mathrm{H}), 1.47-1.48$ (m, $1 \mathrm{H}), 1.69$ (br, $2 \mathrm{H}), 2.70(\mathrm{t}, J=7.7 \mathrm{~Hz}, 2 \mathrm{H}), 7.32(\mathrm{~d}, J=7.9 \mathrm{~Hz}, 2 \mathrm{H}), 7.41(\mathrm{t}, J=3.2 \mathrm{~Hz}, 3 \mathrm{H})$, 7.63-7.58 (m, $4 \mathrm{H}) ;{ }^{13} \mathrm{C} \mathrm{NMR}\left(100 \mathrm{MHz}, \mathrm{CDCl}_{3}\right)$ 8: -3.8, -3.7, 3.5, 9.2, 15.7, 29.5, 31.1, 35.7, $35.9,123.4(\mathrm{q}, J=\mathrm{Hz}), 125.2,127.7,128.2$, 128.9, 133.8, 139.5, 147.0 ppm. HRMS (ESI) calculated for $\mathrm{C}_{22} \mathrm{H}_{28} \mathrm{~F}_{3} \mathrm{Si}(\mathrm{M}+\mathrm{H})^{+} 377.1912$, found 377.1907 .

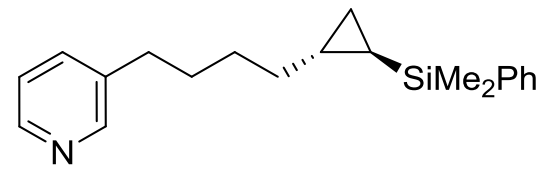

3-(4-((1R,2R)-2-(dimethyl(phenyl)silyl)cyclopropyl)butyl)pyridine (5h):

5h was prepared based on above procedure $B$, purified by flash chromatography (eluting solvents: EtOAc/hexanes $=0-20 \%)$ to give title compound $(\mathrm{X}=\mathrm{Br}, 0.45 \mathrm{~g}$, yield $=77 \%$; and $\mathrm{X}=\mathrm{Cl}, 0.52 \mathrm{~g}$, yield = $63 \%)$ as a colorless oil. ${ }^{1} \mathrm{H} \mathrm{NMR}\left(500 \mathrm{MHz} \mathrm{CDCl}_{3}\right) \delta:-0.44(\mathrm{dt}, J=9.8,6.5 \mathrm{~Hz}, 1 \mathrm{H}), 0.20(\mathrm{~s}, 6$ $\mathrm{H}), 0.43(\mathrm{~d}, J=27.1 \mathrm{~Hz}, 2 \mathrm{H}), 0.69-0.65(\mathrm{~m}, 1 \mathrm{H}), 1.31-1.23(\mathrm{~m}, 1 \mathrm{H}), 1.47-1.40(\mathrm{~m}, 3 \mathrm{H}), 1.68-$ $1.59(\mathrm{~m}, 2 \mathrm{H}), 2.60(\mathrm{t}, J=7.7 \mathrm{~Hz}, 2 \mathrm{H}), 7.21(\mathrm{dd}, J=7.8,4.8 \mathrm{~Hz}, 1 \mathrm{H}), 7.37(\mathrm{t}, J=3.1 \mathrm{~Hz}, 3 \mathrm{H})$, $7.47(\mathrm{~d}, J=7.8 \mathrm{~Hz}, 1 \mathrm{H}), 7.58-7.56(\mathrm{~m}, 2 \mathrm{H}), 8.46(\mathrm{~s}, 2 \mathrm{H}) ;{ }^{13} \mathrm{C} \mathrm{NMR}\left(125 \mathrm{MHz}, \mathrm{CDCl}_{3}\right) \delta:-3.8$, 
-3.7, 3.4, 9.2, 15.6, 29.4, 31.0, 33.1, 35.6, 123.2, 127.7, 128.8, 133.8, 135.8, 137.9, 139.4, 147.2, 150.0 ppm. HRMS (ESI) calculated for $\mathrm{C}_{20} \mathrm{H}_{27} \mathrm{NSi}(\mathrm{M}+\mathrm{H})^{+} 310.1998$, found 310.1986 .

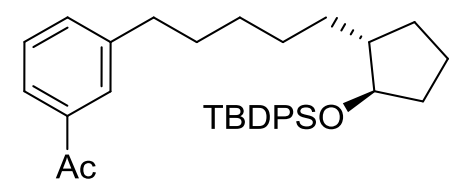

1-(3-(5-((1R,2R)-2-((tert-Butyldiphenylsilyl)oxy)cyclopentyl)pentyl)phenyl)ethan-1-one (5i):

5i was prepared based on above procedure $B$, purified by flash chromatography (eluting solvents: EtOAc/hexanes $=0-10 \%)$ to give title compound $(\mathrm{X}=\mathrm{Br}, 0.64 \mathrm{~g}$, yield $=83 \%$; and $\mathrm{X}=\mathrm{Cl}, 0.81 \mathrm{~g}$, yield $=$ 81\%) as a colorless oil. ${ }^{1} \mathrm{H}$ NMR $\left(500 \mathrm{MHz}, \mathrm{CDCl}_{3}\right) \delta$ : 0.94-0.85 (m, $\left.1 \mathrm{H}\right), 1.07(\mathrm{~m}, 11 \mathrm{H}), 1.22$ $(\mathrm{m}, 5 \mathrm{H}), 1.50-1.45(\mathrm{~m}, 1 \mathrm{H}), 1.62-1.54(\mathrm{~m}, 4 \mathrm{H}), 1.76-1.70(\mathrm{~m}, 1 \mathrm{H}), 1.82(\mathrm{t}, J=7.5 \mathrm{~Hz}, 1 \mathrm{H})$, 1.93-1.87 (m, $1 \mathrm{H}), 2.64-2.61(\mathrm{~m}, 5 \mathrm{H}), 3.85(\mathrm{q}, J=4.9 \mathrm{~Hz}, 1 \mathrm{H}), 7.42-7.36(\mathrm{~m}, 8 \mathrm{H}), 7.69-7.67$ (m, $4 \mathrm{H}), 7.80-7.78$ (m, $2 \mathrm{H}) ;{ }^{13} \mathrm{C}$ NMR $\left(125 \mathrm{MHz}, \mathrm{CDCl}_{3}\right)$ \%: 19.2, 22.0, 26.7, 27.1, 27.8, 29.4, 31.3, 33.5, 34.4, 35.8, 48.2, 80.4, 125.9, 127.4, 128.1, 128.4, 129.41, 129.43, 133.3, 134.8, 134.9, 135.90, 135.92, 137.2, 143.5, 198.4 ppm. HRMS (ESI) calculated for $\mathrm{C}_{34} \mathrm{H}_{44} \mathrm{O}_{2} \mathrm{Si}(\mathrm{M}+\mathrm{H})^{+}$ 513.3184, found 513.3183.<smiles>[13CH2]OC1CCCC1CCCCCc1cnc2ccccc2c1</smiles>

3-(5-((1R,2R)-2-((tert-Butyldiphenylsilyl)oxy)cyclopentyl)pentyl)quinoline (5j):

5j was prepared based on above procedure B, purified by flash chromatography (eluting solvents: EtOAc/hexanes $=0-20 \%$ ) to give title compound $(0.56 \mathrm{~g}$, yield $=75 \%)$ as a colorless oil. ${ }^{1} \mathrm{H}$ NMR $(500$ $\left.\mathrm{MHz}, \mathrm{CDCl}_{3}\right) \delta: 0.88(\mathrm{t}, J=8.7 \mathrm{~Hz}, 1 \mathrm{H}), 1.04(\mathrm{~s}, 9 \mathrm{H}), 1.20-1.30(\mathrm{~m}, 5 \mathrm{H}), 1.47(\mathrm{~m}, 1 \mathrm{H}), 1.65-$ $1.51(\mathrm{~m}, 5 \mathrm{H}), 1.70-1.67(\mathrm{~m}, 1 \mathrm{H}), 1.84-1.80(\mathrm{~m}, 1 \mathrm{H}), 1.90-1.87(\mathrm{~m}, 1 \mathrm{H}), 2.74(\mathrm{t}, J=7.7 \mathrm{~Hz}, 2$ $\mathrm{H}), 3.82(\mathrm{~d}, J=5.4 \mathrm{~Hz}, 1 \mathrm{H}), 7.39-7.34(\mathrm{~m}, 6 \mathrm{H}), 7.53(\mathrm{t}, J=7.5 \mathrm{~Hz}, 1 \mathrm{H}), 7.66(\mathrm{~d}, J=5.8 \mathrm{~Hz}, 5$ H), $7.77(\mathrm{~d}, J=8.1 \mathrm{~Hz}, 1 \mathrm{H}), 7.88(\mathrm{~s}, 1 \mathrm{H}), 8.09(\mathrm{~d}, J=8.4 \mathrm{~Hz}, 1 \mathrm{H}), 8.76(\mathrm{~s}, 1 \mathrm{H}) ;{ }^{13} \mathrm{C}$ NMR $\left(125 \mathrm{MHz}, \mathrm{CDCl}_{3}\right)$ 8: 19.2, 22.0, 27.1, 27.8, 29.3, 29.4, 31.0, 33.2, 33.5, 34.4, 48.2, 80.4, 126.5, 127.3, 127.4, 128.2, 128.5, 129.2, 129.42, 129.43, 134.1, 134.76, 134.84, 135.4, 135.9, 146.8, 152.2 ppm. HRMS (ESI) calculated for $\mathrm{C}_{35} \mathrm{H}_{43} \mathrm{NOSi}(\mathrm{M}+\mathrm{H})^{+} 522.3195$, found 522.3187.

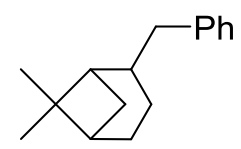

2-Benzyl-6,6-dimethylbicyclo[3.1.1]heptane (5k):

5k was prepared based on above procedure $\mathrm{B}$, purified by flash chromatography (eluting solvent: hexanes) to give title compound $(0.30 \mathrm{~g}$, yield $=74 \%)$ as a colorless oil. ${ }^{1} \mathrm{H} N \mathrm{NR}\left(500 \mathrm{MHz}, \mathrm{CDCl}_{3}\right) \delta$ : $0.90(\mathrm{~d}, J=9.6 \mathrm{~Hz}, 1 \mathrm{H}), 1.20(\mathrm{~s}, 3 \mathrm{H}), 1.25(\mathrm{~s}, 3 \mathrm{H}), 1.65-1.56(\mathrm{~m}, 1 \mathrm{H}), 2.01-1.87(\mathrm{~m}, 5 \mathrm{H})$, $2.36(\mathrm{~d}, J=9.7 \mathrm{~Hz}, 2 \mathrm{H}), 2.78-2.69(\mathrm{~m}, 2 \mathrm{H}), 7.23-7.19(\mathrm{~m}, 3 \mathrm{H}), 7.35-7.30(\mathrm{~m}, 2 \mathrm{H}) ;{ }^{13} \mathrm{C} \mathrm{NMR}$ 
$\left(125 \mathrm{MHz}, \mathrm{CDCl}_{3}\right) \delta: 22.4,23.5,26.5,28.3,33.8,38.8,41.6,43.3,43.5,45.3,125.6,128.1$, 129.1, 142.0 ppm. HRMS (ESI) calculated for $\mathrm{C}_{16} \mathrm{H}_{22}(\mathrm{M})^{+} 214.1742$, found 214.1742.

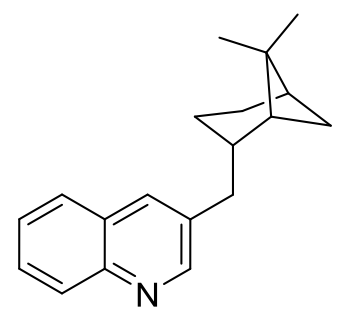

3-(((1R,2S,5R)-6,6-Dimethylbicyclo[3.1.1] heptan-2-yl)methyl)quinoline (5l):

5I was prepared based on above procedure B, purified by flash chromatography (eluting solvents: EtOAc/hexanes $=0-20 \%)$ to give title compound $(0.31 \mathrm{~g}$, yield $=82 \%$; or $0.32 \mathrm{~g}$, yield $=84 \%$ by RuPhos as ligand) as a colorless oil. ${ }^{1} \mathrm{H} \mathrm{NMR}\left(500 \mathrm{MHz}^{\left.-\mathrm{CDCl}_{3}\right)} \delta: 0.87(\mathrm{~d}, J=9.6 \mathrm{~Hz}, 1 \mathrm{H}), 1.19(\mathrm{~s}, 3 \mathrm{H})\right.$, $1.21(\mathrm{~s}, 3 \mathrm{H}), 1.64-1.59(\mathrm{~m}, 1 \mathrm{H}), 1.93-1.84(\mathrm{~m}, 4 \mathrm{H}), 1.99(\mathrm{t}, J=12.2 \mathrm{~Hz}, 1 \mathrm{H}) ; 2.33-2.29$ (m, 1 H), $2.42(\mathrm{t}, J=8.6 \mathrm{~Hz}, 1 \mathrm{H}), 2.90-2.81(\mathrm{~m}, 2 \mathrm{H}), 7.51(\mathrm{t}, J=7.5 \mathrm{~Hz}, 1 \mathrm{H}), 7.64(\mathrm{t}, J=7.5 \mathrm{~Hz}, 1$ $\mathrm{H}), 7.76(\mathrm{~d}, J=8.2 \mathrm{~Hz}, 1 \mathrm{H}), 7.87(\mathrm{~s}, 1 \mathrm{H}), 8.08(\mathrm{~d}, J=8.4 \mathrm{~Hz}, 1 \mathrm{H}), 8.76(\mathrm{~s}, 1 \mathrm{H}) ;{ }^{13} \mathrm{C}$ NMR

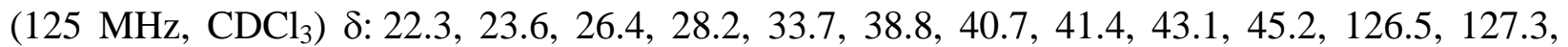
128.5, 129.2, 134.8, 146.8, 152.5 ppm. HRMS (ESI) calculated for $\mathrm{C}_{19} \mathrm{H}_{23} \mathrm{~N}(\mathrm{M}+\mathrm{H})^{+} 266.1915$, found 266.1903 .
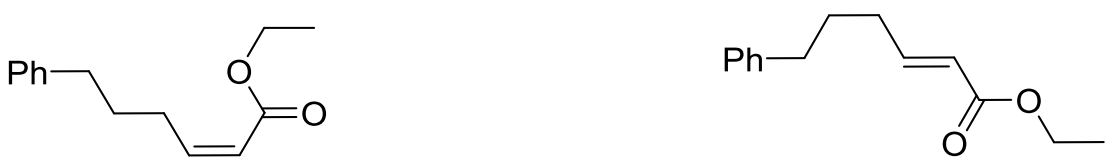

Ethyl (Z)-6-phenylhex-2-enoate (5m-cis) and Ethyl (E)-6-phenylhex-2-enoate (5m-trans)

Both of $\mathbf{5 m}$-cis and $\mathbf{5 m}$-trans were prepared based on above procedure $B$ through the coupling reaction of ethyl (Z)-3-bromoacrylate with tris(3-phenylpropyl)borane to give cis:trans $=9: 1$. The mixture was separated by flash chromatography (eluting solvents: $\mathrm{MTBE} /$ hexanes $=0-5 \%$ ) to give title compounds $5 \mathrm{~m}$-cis $(0.19 \mathrm{~g})$, yield $=52 \%$ as a colorless oil. ${ }^{1} \mathrm{H}$ NMR $\left(400 \mathrm{MHz}, \mathrm{CDCl}_{3}\right) \delta: 1.28(\mathrm{t}, J=7.1 \mathrm{~Hz}, 3$ $\mathrm{H}), 1.82-1.74(\mathrm{~m}, 2 \mathrm{H}), 2.74-2.64(\mathrm{~m}, 4 \mathrm{H}), 4.16$ (q, $J=7.1 \mathrm{~Hz}, 2 \mathrm{H}), 5.80$ (dt, $J=11.6,1.8 \mathrm{~Hz}, 1$ $\mathrm{H}), 6.23(\mathrm{dt}, J=11.6,7.6 \mathrm{~Hz}, 1 \mathrm{H}), 7.20-7.16(\mathrm{~m}, 3 \mathrm{H}), 7.30-7.25(\mathrm{~m}, 2 \mathrm{H}), ;{ }^{13} \mathrm{C} \mathrm{NMR}(100$ $\mathrm{MHz}, \mathrm{CDC13}) \delta: 14.2,28.7,30.8,35.6,59.8,120.1,125.8,128.3,128.4,142.1,149.8,166.4$ ppm. HRMS (ESI) calculated for $\mathrm{C}_{14} \mathrm{H}_{19} \mathrm{O}_{2}(\mathrm{M}+\mathrm{H})^{+} 219.1385$, found 219.1386. 5m-trans (0.018 $\mathrm{g})$, yield $=5 \%$ as a colorless oil. ${ }^{1} \mathrm{H} \mathrm{NMR}\left(400 \mathrm{MHz}, \mathrm{CDCl}_{3}\right) \delta: 1.28(\mathrm{t}, J=7.1 \mathrm{~Hz}, 3 \mathrm{H}), 1.80(\mathrm{~m}, 2$ H), $2.34(\mathrm{~m}, 2 \mathrm{H}), 2.65(\mathrm{t}, J=7.6 \mathrm{~Hz}, 2 \mathrm{H}), 4.19(\mathrm{q}, J=7.1 \mathrm{~Hz}, 2 \mathrm{H}), 5.83(\mathrm{dt}, J=15.6,1.6 \mathrm{~Hz}, 1$ $\mathrm{H}), 6.98(\mathrm{dt}, J=15.6,7.0 \mathrm{~Hz}, 1 \mathrm{H}), 7.21-7.16(\mathrm{~m}, 3 \mathrm{H}), 7.30-7.25(\mathrm{~m}, 2 \mathrm{H}) ;{ }^{13} \mathrm{C} \mathrm{NMR}(100$ $\mathrm{MHz}, \mathrm{CDCl3}) \delta: 14.2,29.6,31.6,35.2,60.2,121.7,125.9,128.3,128.4,141.7,148.7,166.7$ ppm. HRMS (ESI) calculated for $\mathrm{C}_{14} \mathrm{H}_{19} \mathrm{O}_{2}(\mathrm{M}+\mathrm{H})^{+} 219.1385$, found 219.1396 .

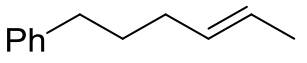


(E)-Hex-4-en-1-ylbenzene (5n)

5n was prepared based on above procedure $B$ through the coupling reaction of (E)-1-bromoprop-1-ene with tris(3-phenylpropyl)borane, purified by flash chromatography (eluting solvent: hexanes) to give title compound $(0.24 \mathrm{~g}$, yield $=60 \%)$ as a colorless oil. ${ }^{1} \mathrm{H} \mathrm{NMR}\left(400 \mathrm{MHz}, \mathrm{CDCl}_{3}\right) \delta$ : 1.74-1.67 (m, $\left.5 \mathrm{H}\right)$, 2.07-2.02 (m, $2 \mathrm{H}), 2.63(\mathrm{t}, J=7.7 \mathrm{~Hz}, 2 \mathrm{H}), 5.19(\mathrm{~m}, 2 \mathrm{H}), 7.22-7.18(\mathrm{~m}, 3 \mathrm{H}), 7.31-7.28(\mathrm{~m}, 2$ $\mathrm{H}) ;{ }^{13} \mathrm{C}$ NMR (100 MHz, CDCl3) $\delta: 17.9,31.3,32.1,35.4,125.2,125.6,128.2,128.4,131.0$, 142.6 ppm. HRMS (ESI) calculated for $\mathrm{C}_{12} \mathrm{H}_{17}(\mathrm{M}+\mathrm{H})^{+} 161.1330$, found 161.1340.

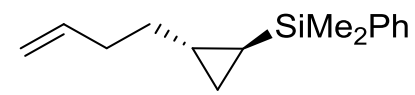

((1S,2S)-2-(But-3-en-1-yl)cyclopropyl)dimethyl(phenyl)silane:

${ }^{1} \mathrm{H}$ NMR (500 MHz, $\left.\mathrm{CDCl}_{3}\right) \delta:-0.40(\mathrm{dt}, J=9.8,6.6 \mathrm{~Hz}, 1 \mathrm{H}), 0.21(\mathrm{~s}, 3 \mathrm{H}), 0.24(\mathrm{~s}, 3 \mathrm{H}), 0.46$ $(\mathrm{d}, J=14.0 \mathrm{~Hz}, 2 \mathrm{H}), 0.74-0.69(\mathrm{~m}, 1 \mathrm{H}), 1.35-1.26(\mathrm{~m}, 1 \mathrm{H}), 1.56-1.49(\mathrm{~m}, 1 \mathrm{H}), 2.18(\mathrm{q}, J=7.3$ $\mathrm{Hz}, 2 \mathrm{H}), 4.97$ (d, $J=10.2 \mathrm{~Hz}, 1 \mathrm{H}), 5.05$ (t, $J=17.1 \mathrm{~Hz}, 1 \mathrm{H}), 5.88$ (ddt, $J=17.3,10.2,6.5 \mathrm{~Hz}$, $1 \mathrm{H}), 7.39(\mathrm{t}, J=3.2 \mathrm{~Hz}, 3 \mathrm{H}), 7.60-7.58(\mathrm{~m}, 2 \mathrm{H}) ;{ }^{13} \mathrm{C} \mathrm{NMR}\left(125 \mathrm{MHz}, \mathrm{CDCl}_{3}\right) \delta$ : $-3.9,-3.6$, $3.5,9.1,15.5,34.1,35.4,114.1,114.2,127.7,128.8,133.8,139.0,139.4$ ppm. HRMS (ESI) calculated for $\mathrm{C}_{15} \mathrm{H}_{23} \mathrm{Si}(\mathrm{M}+\mathrm{H})^{+} 231.1569$, found 231.1565 .

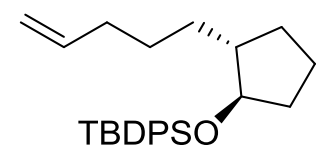

tert-Butyl(((1R,2R)-2-(pent-4-en-1-yl)cyclopentyl)oxy)diphenylsilane:

${ }^{1} \mathrm{H}$ NMR $\left(500 \mathrm{MHz}, \mathrm{CDCl}_{3}\right) \delta: 0.95-0.88(\mathrm{~m}, 1 \mathrm{H}), 1.08(\mathrm{~m}, 11 \mathrm{H}), 1.28(\mathrm{~m}, 3 \mathrm{H}), 1.61-1.46(\mathrm{~m}$, $2 \mathrm{H}), 1.76-1.69(\mathrm{~m}, 1 \mathrm{H}), 1.85-1.83(\mathrm{~m}, 1 \mathrm{H}), 1.99-1.86(\mathrm{~m}, 3 \mathrm{H}), 3.86(\mathrm{q}, J=5.03 \mathrm{~Hz}, 1 \mathrm{H})$, 4.97-4.90 (m, $2 \mathrm{H}), 5.75$ (ddt, $J=17.1, ; 10.2,6.7 \mathrm{~Hz}, 1 \mathrm{H}), 7.42-7.34$ (m, $6 \mathrm{H}), 7.71-7.68$ (m, 4 $\mathrm{H}) ;{ }^{13} \mathrm{C}$ NMR $\left(125 \mathrm{MHz}, \mathrm{CDCl}_{3}\right) \delta: 19.2,22.0,27.1,27.3,29.3,33.1,33.9,34.4,48.1,80.4$, 114.1, 127.5, 127.6, 129.4, 134.7, 134.9, 135.6, 135.9, 139.1 ppm. HRMS (ESI) calculated for $\mathrm{C}_{15} \mathrm{H}_{22} \mathrm{Si}(\mathrm{M})^{+} 230.1487$, found 230.1490 . 


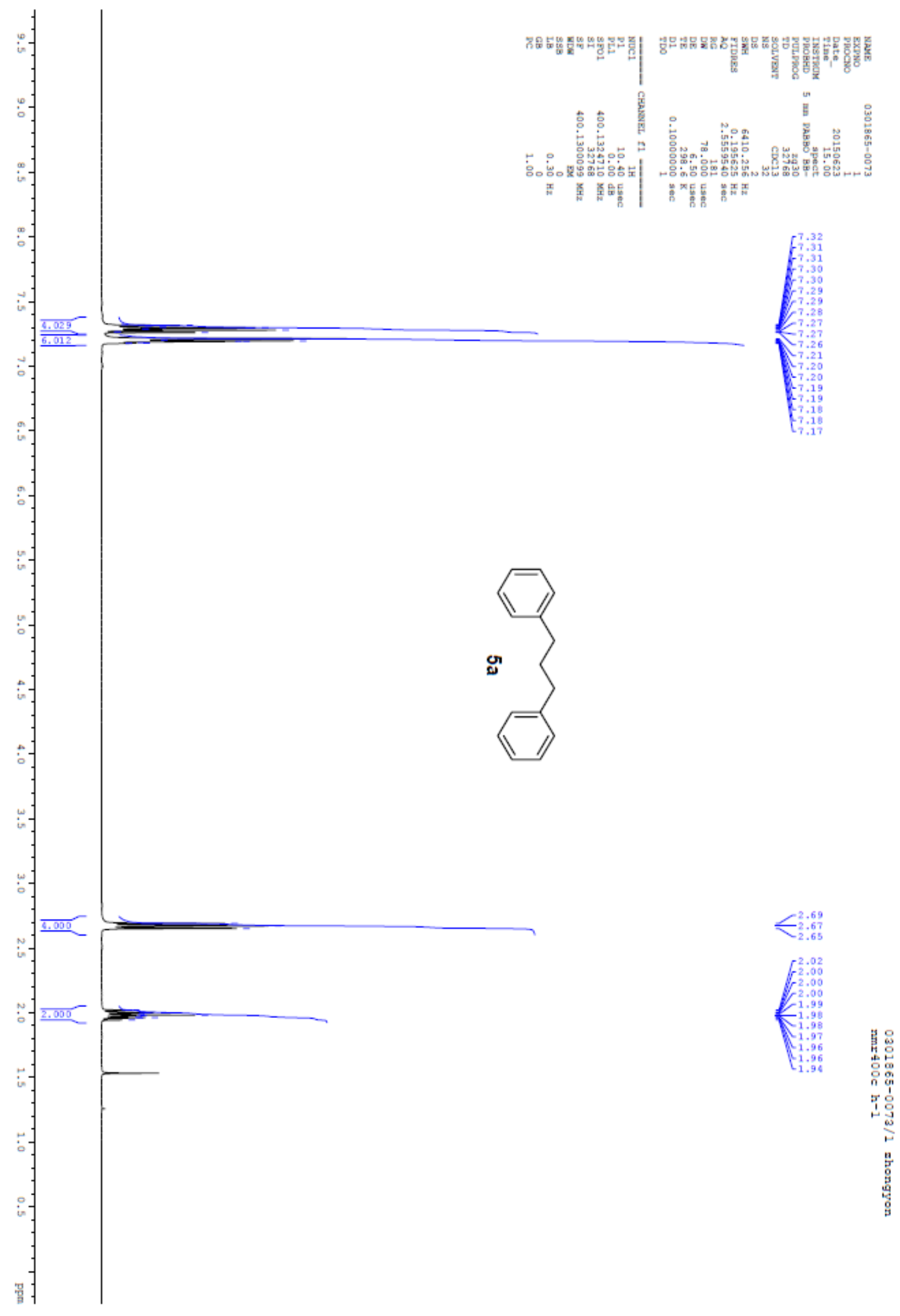




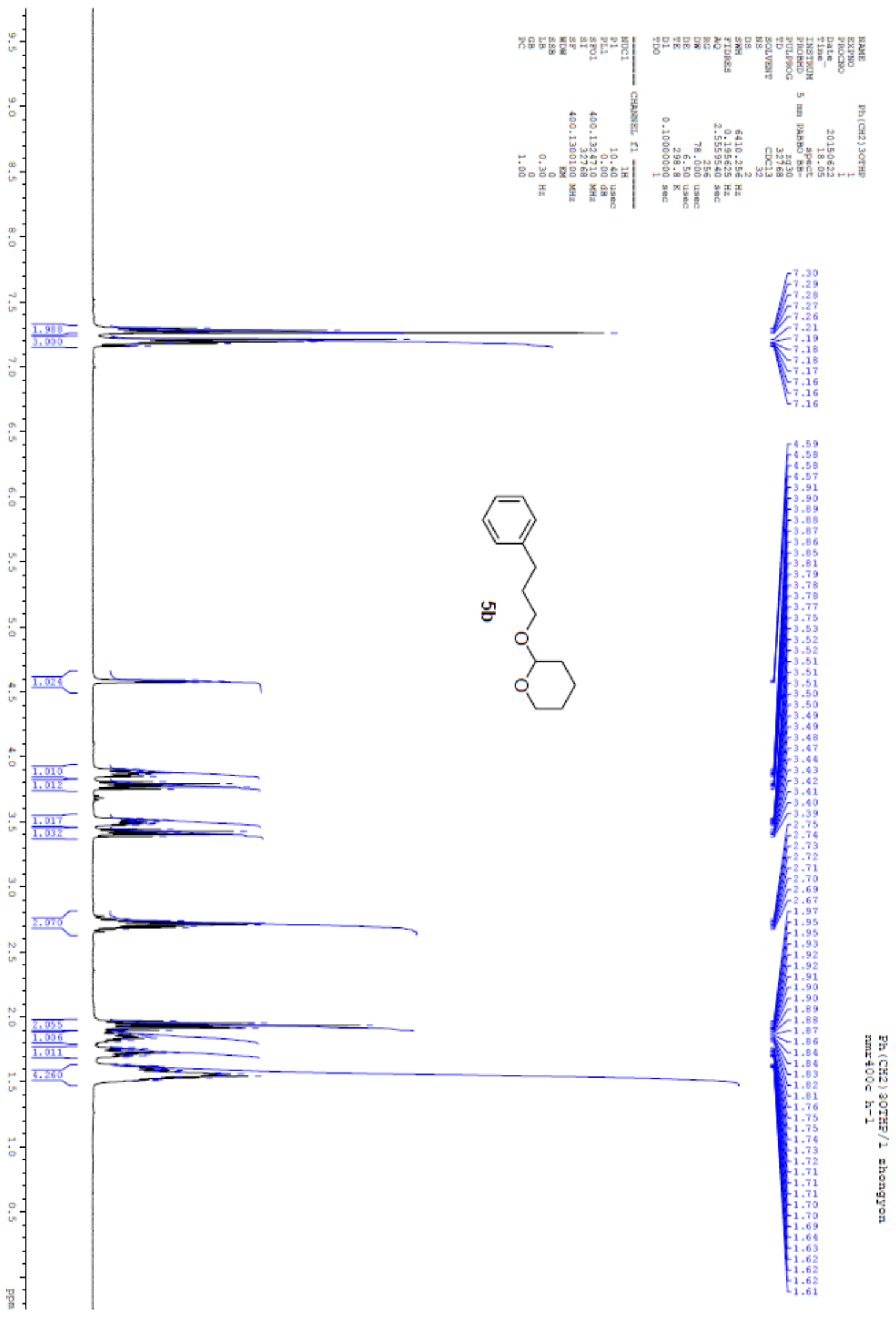




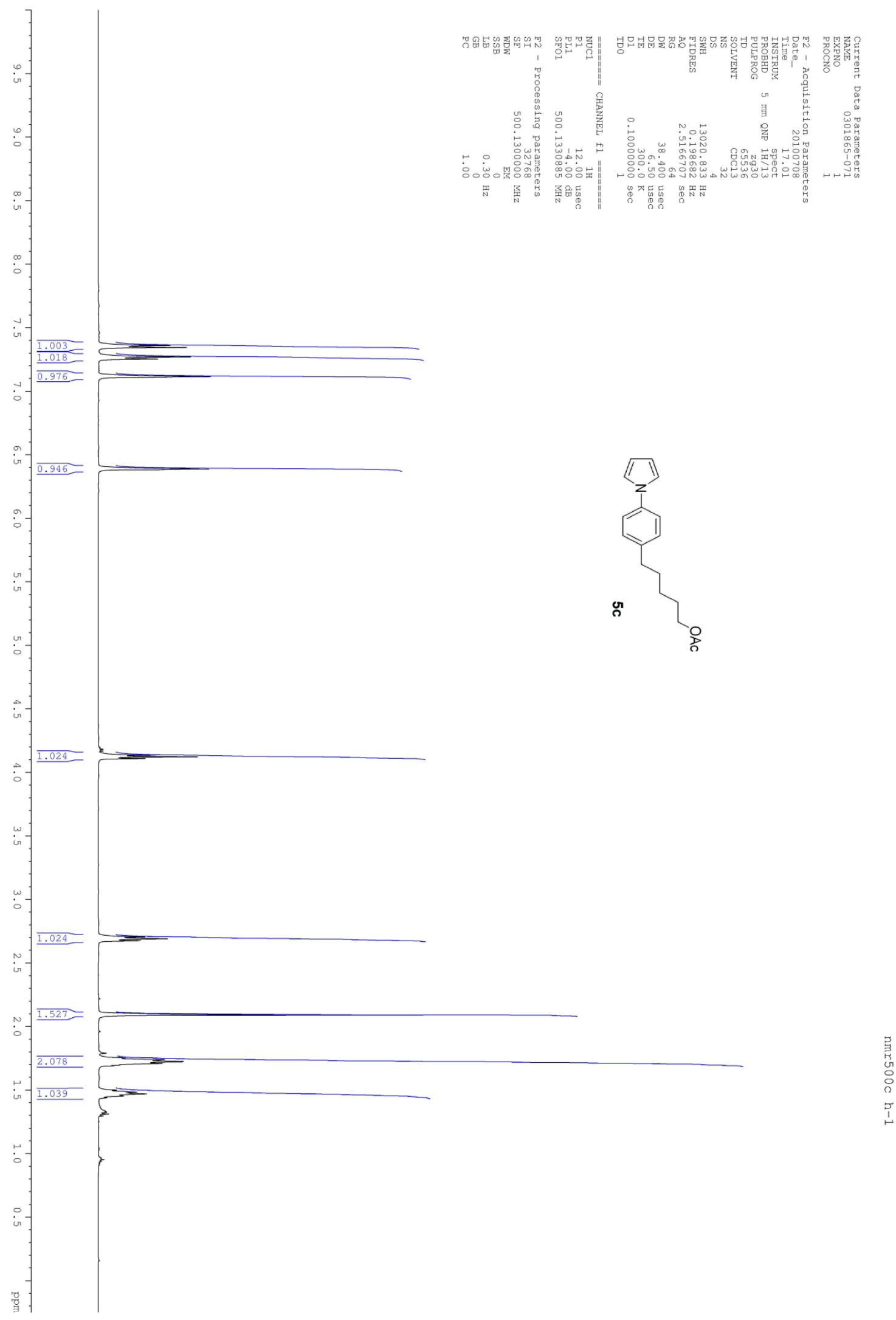



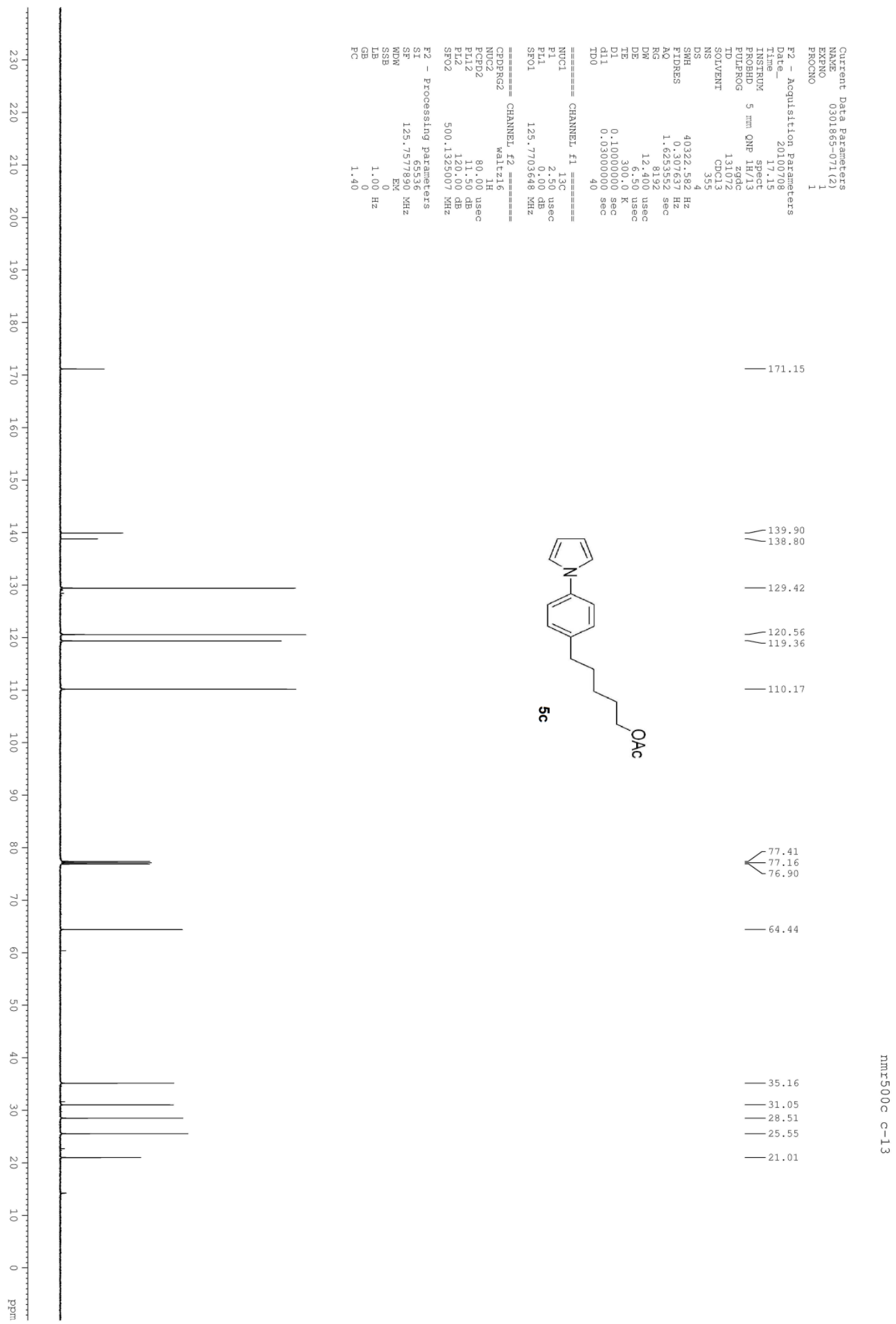


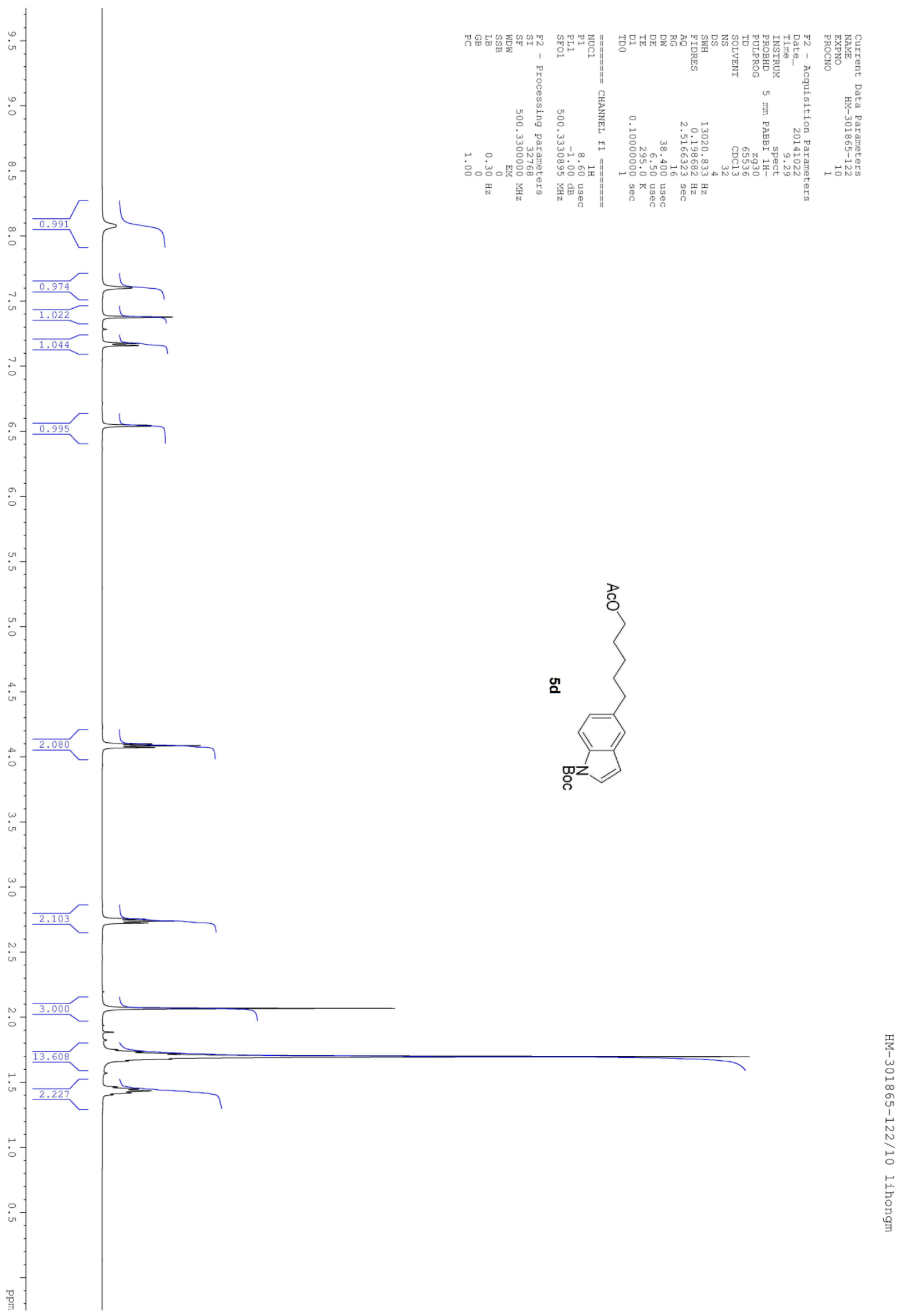




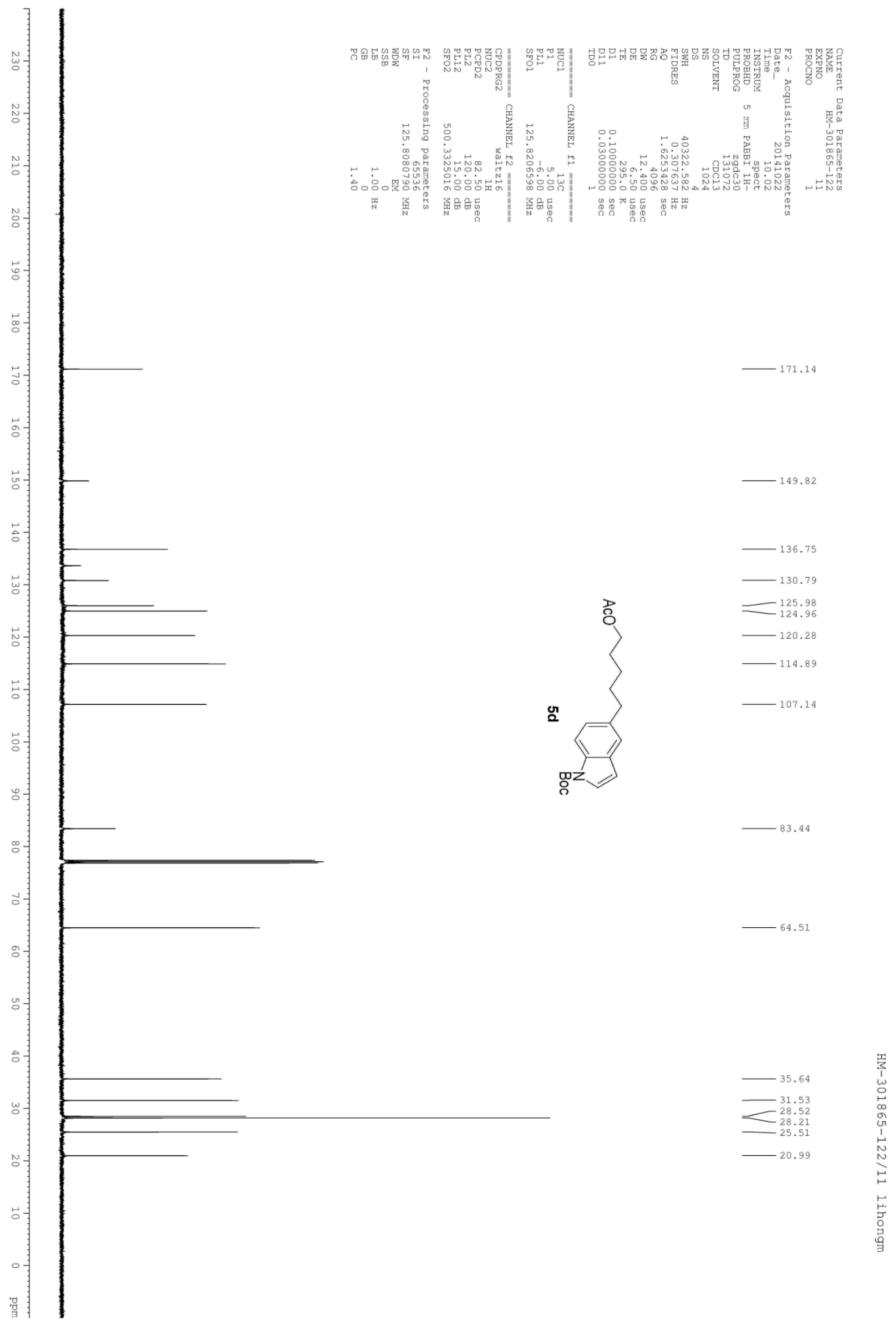




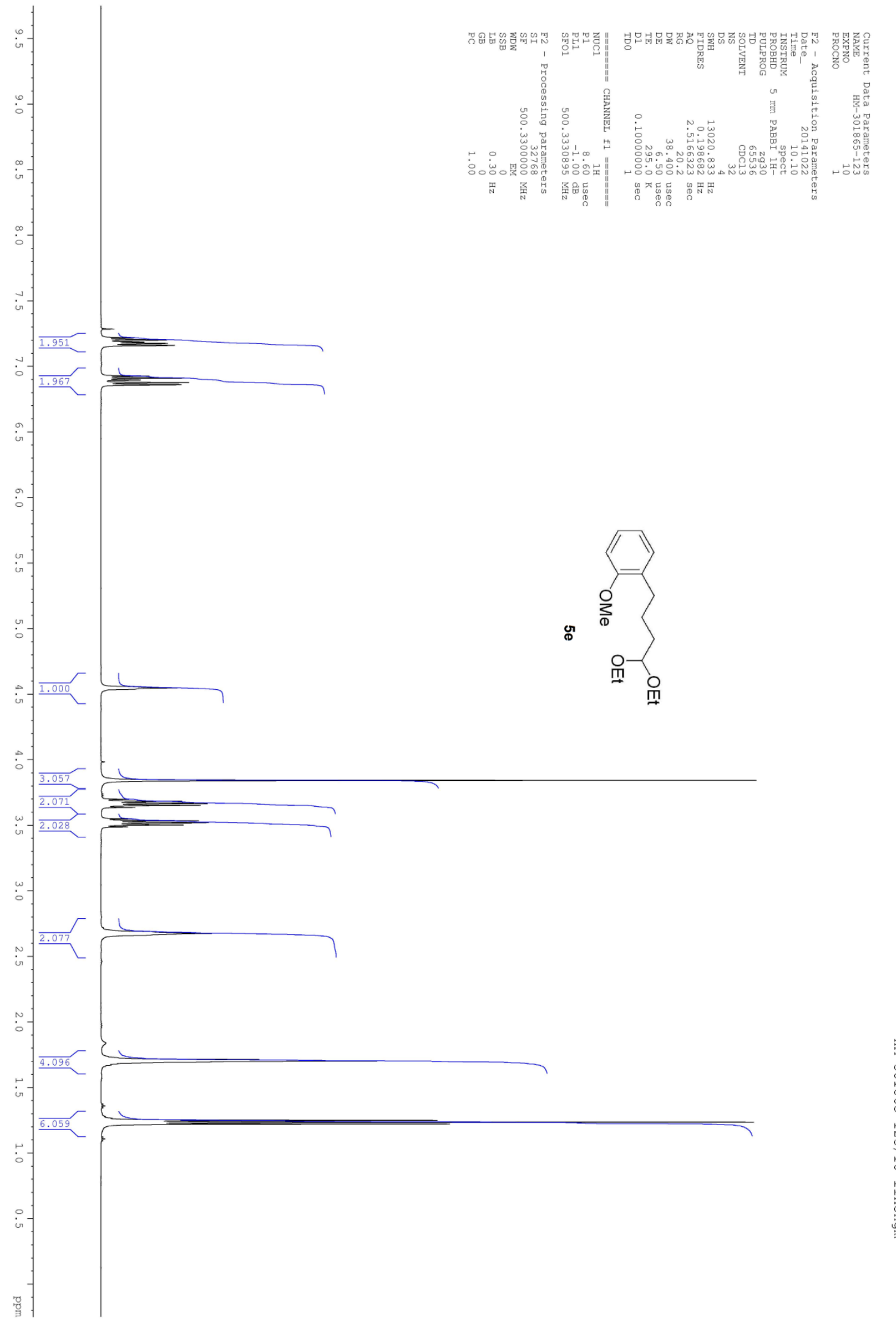




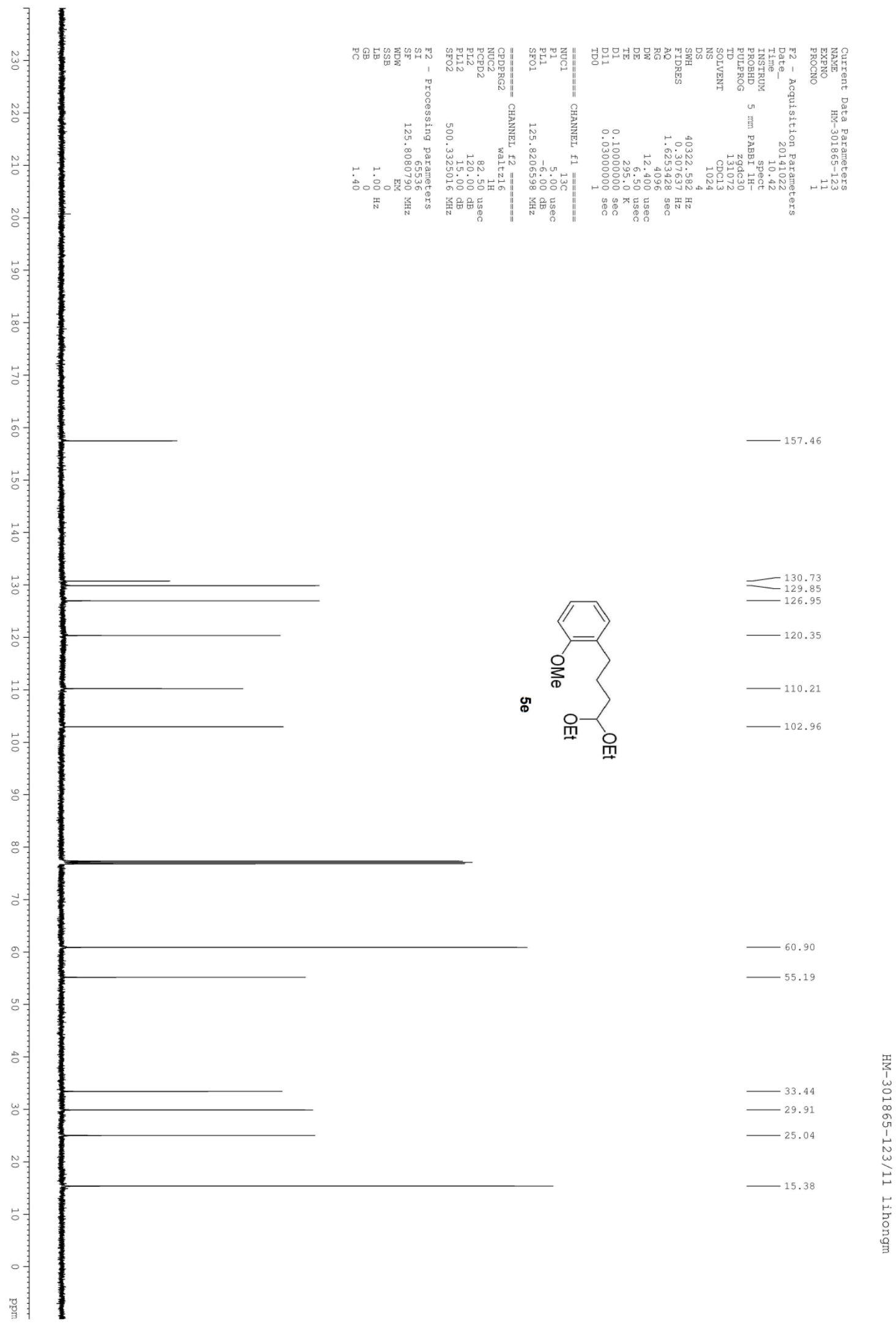




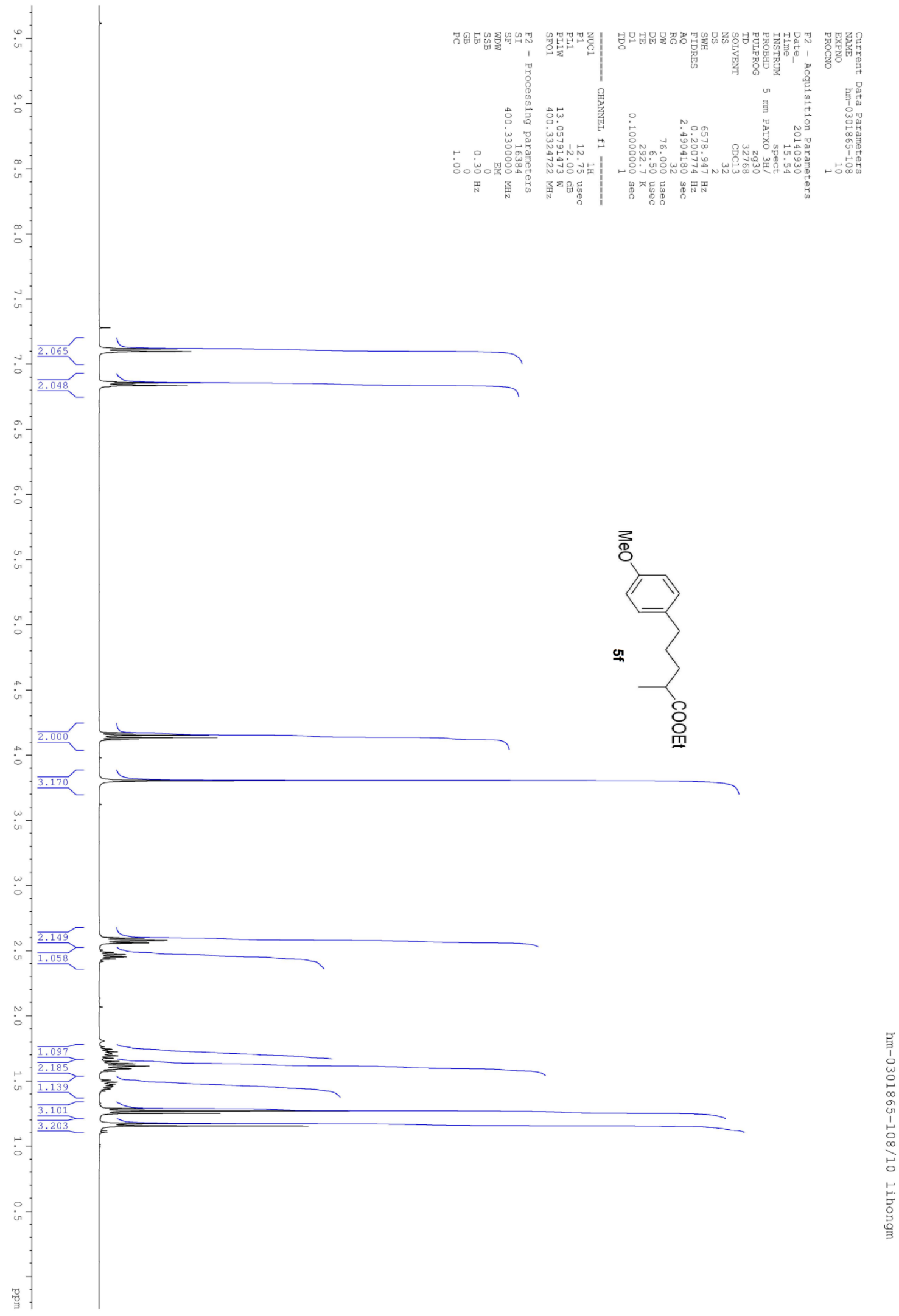




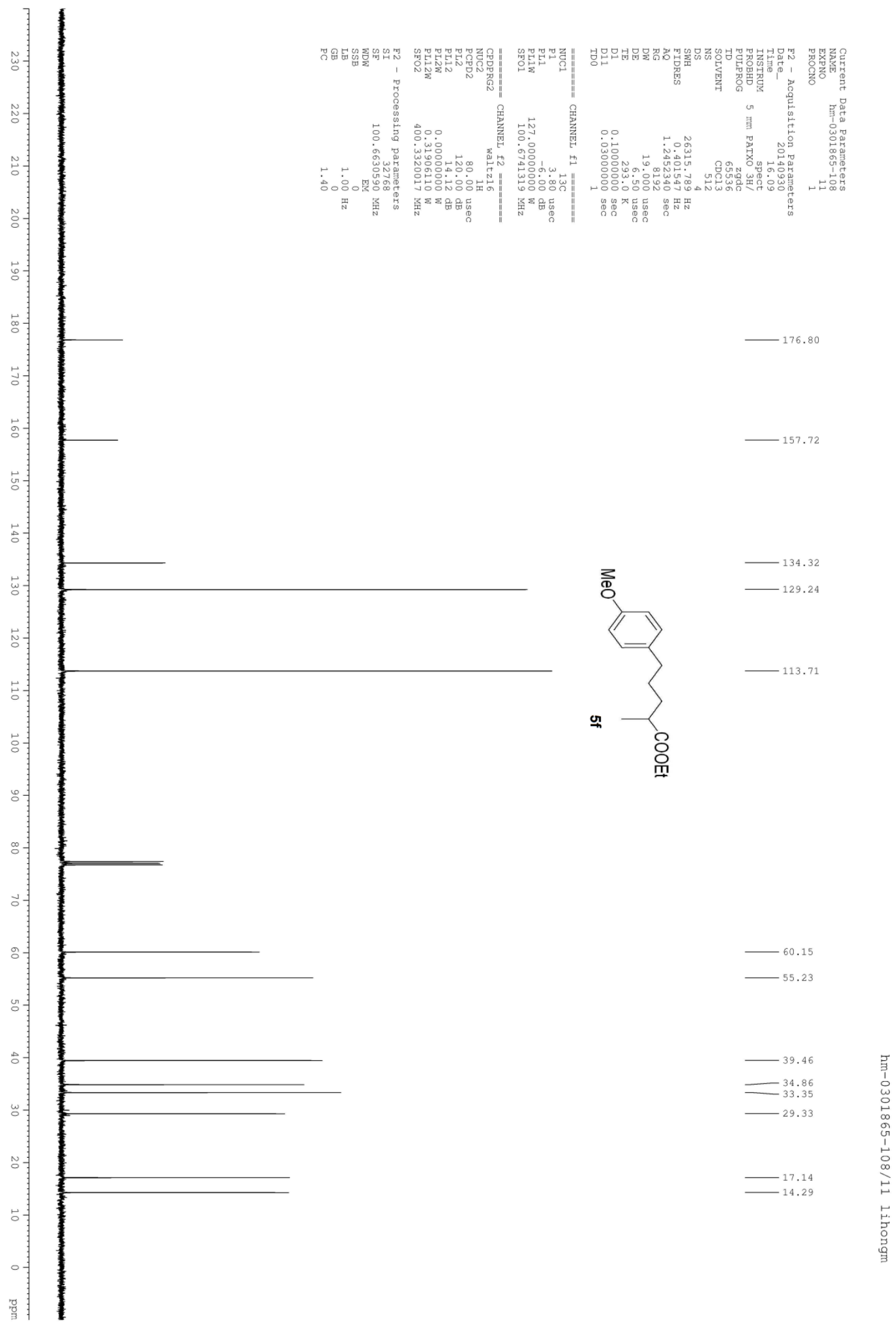




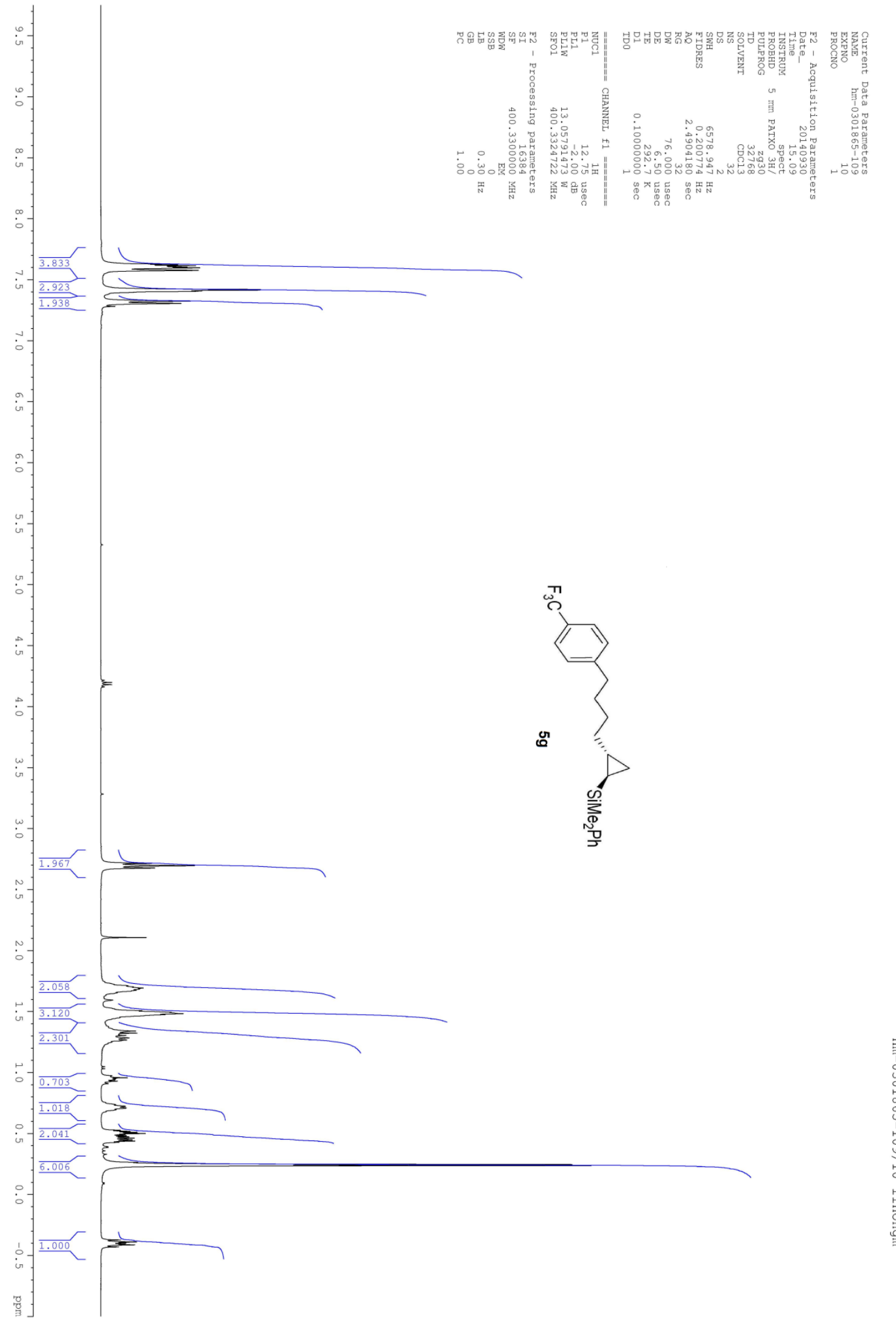



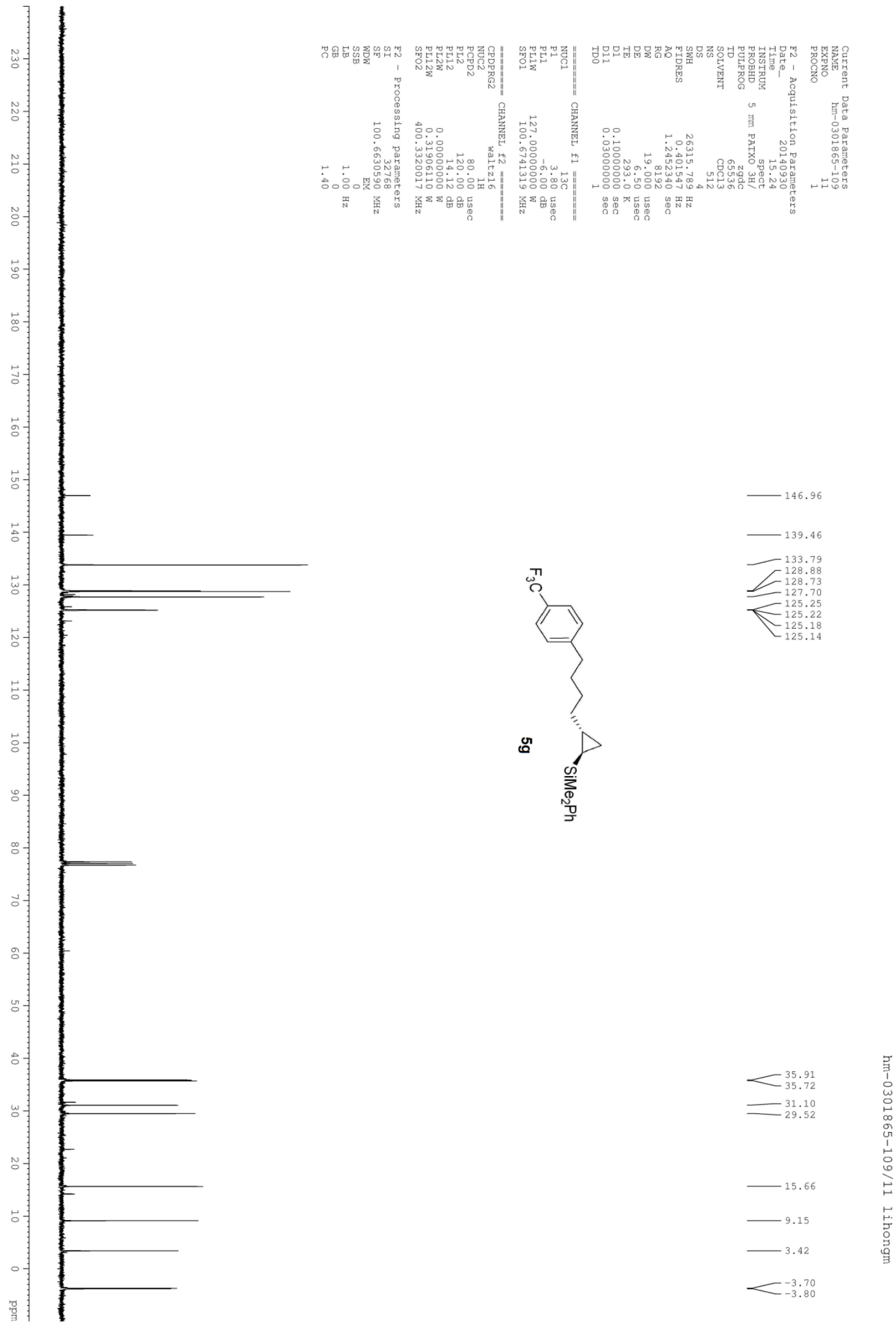

-3.70
-3.80 


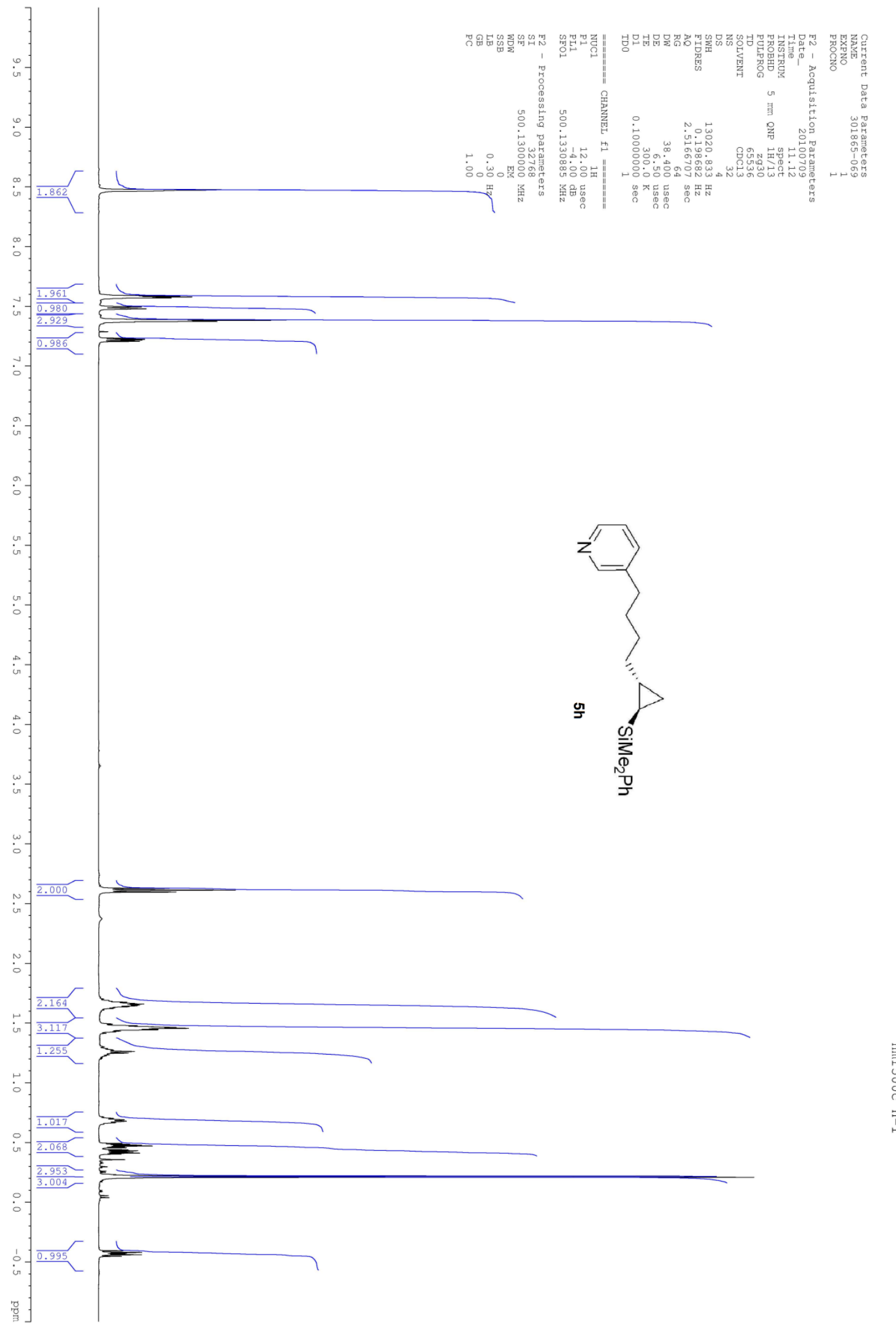



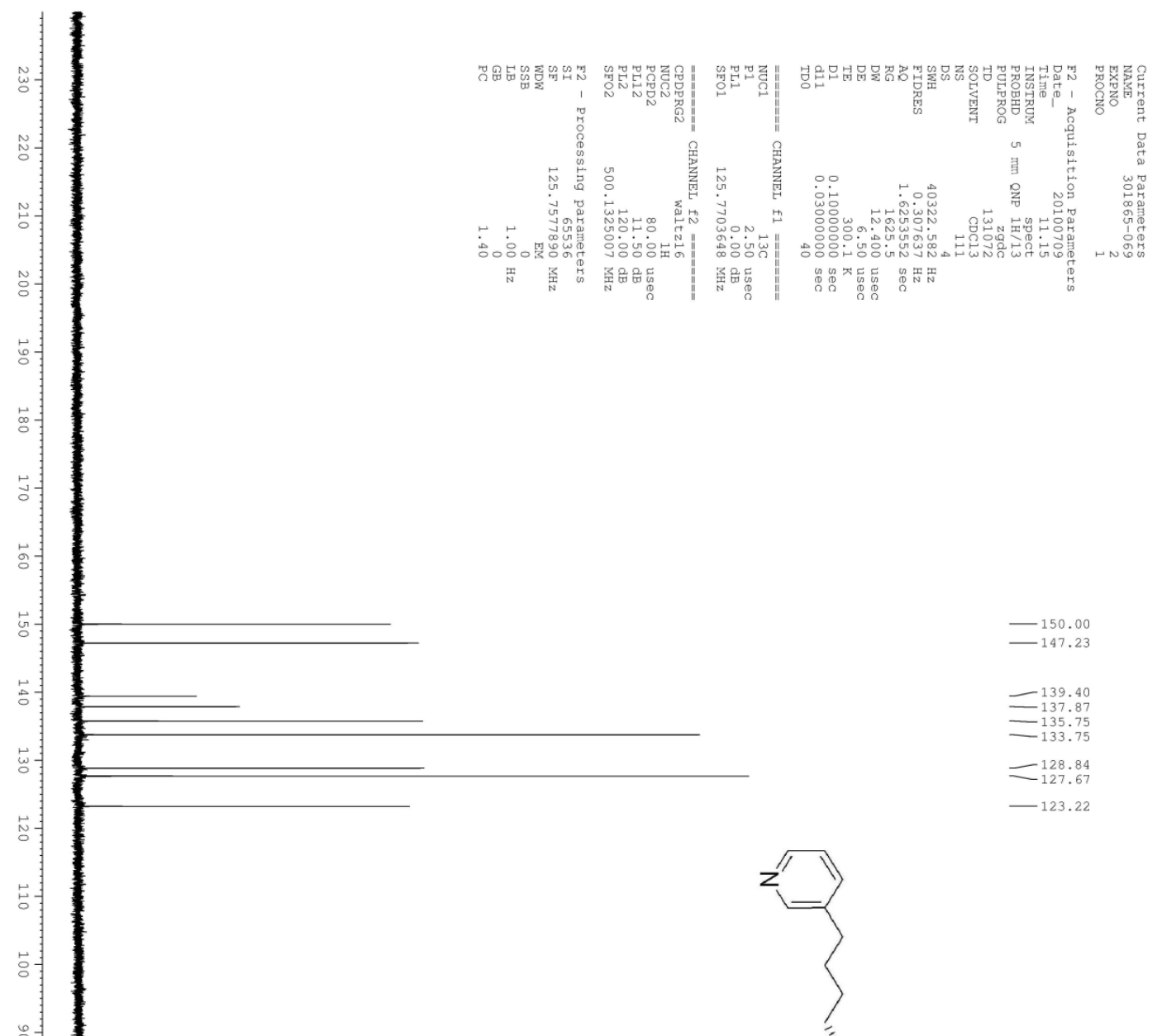

의
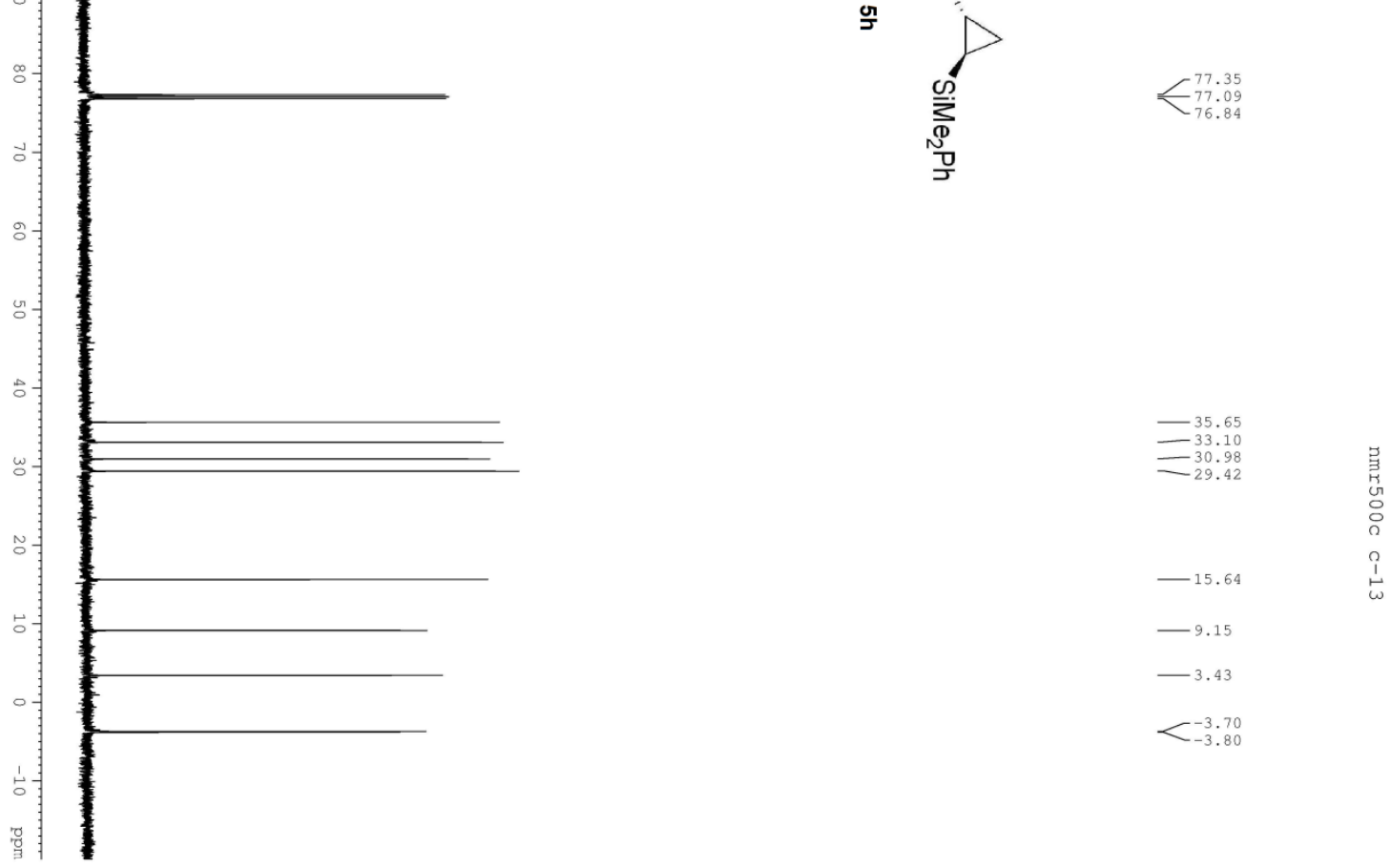


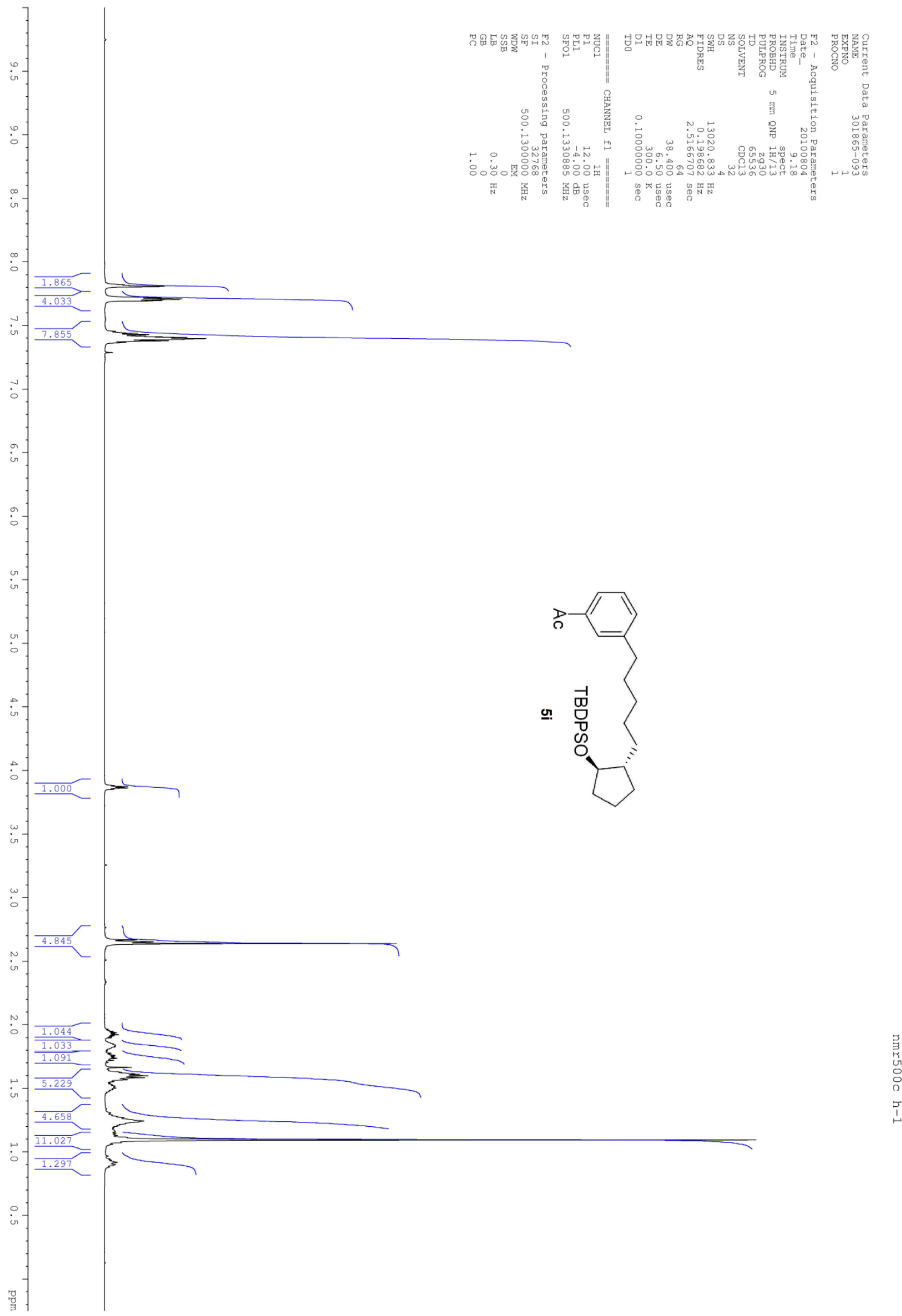




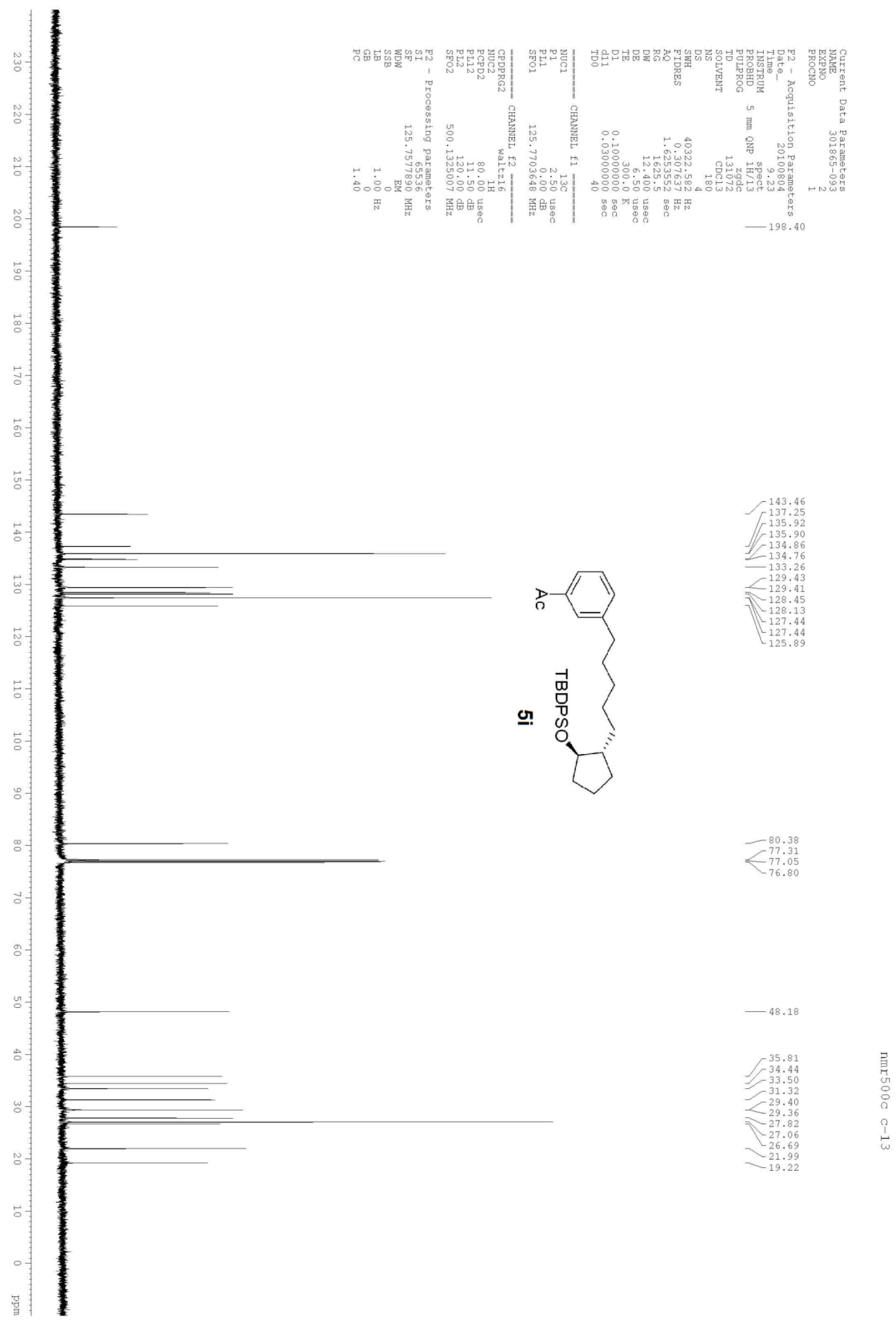




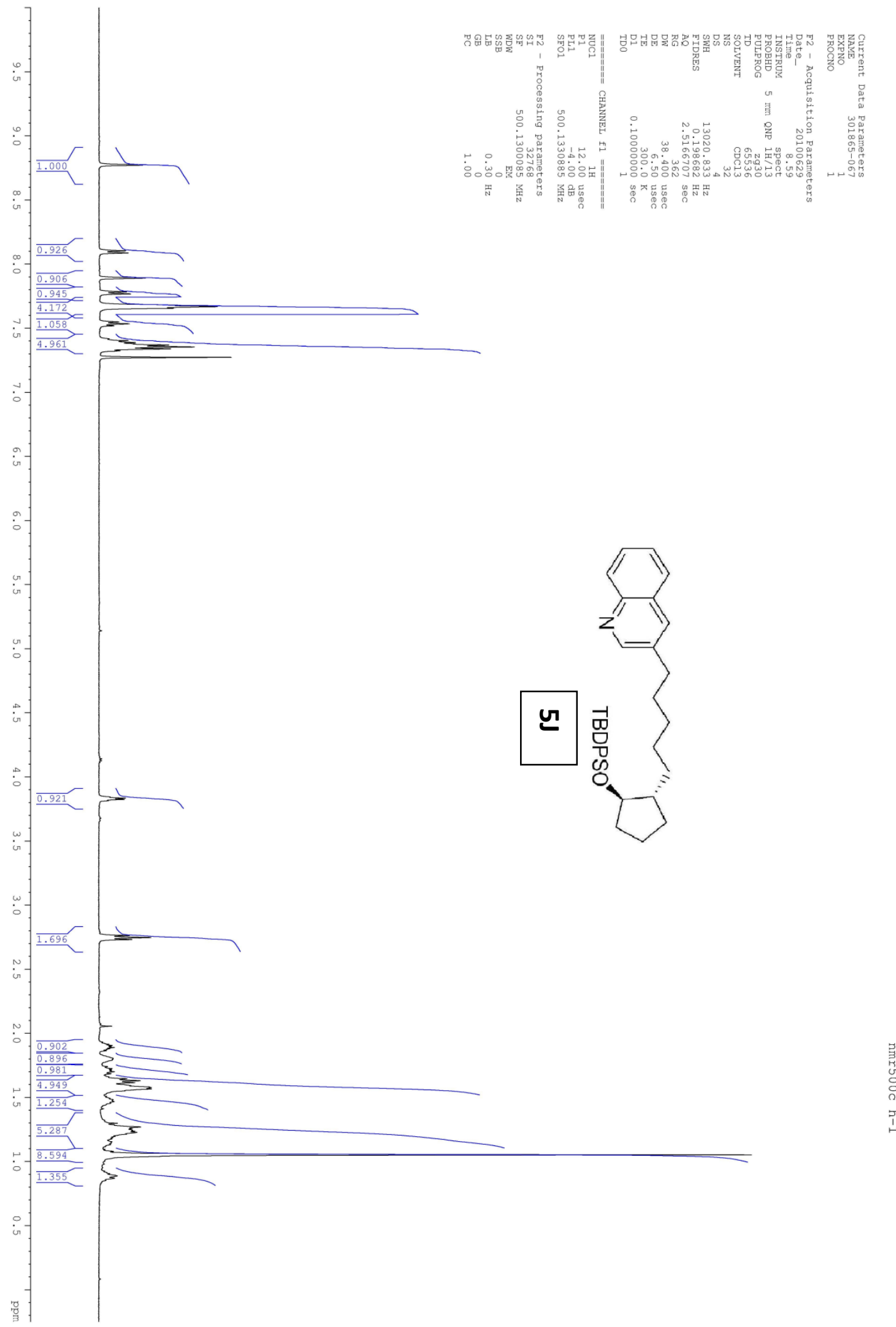




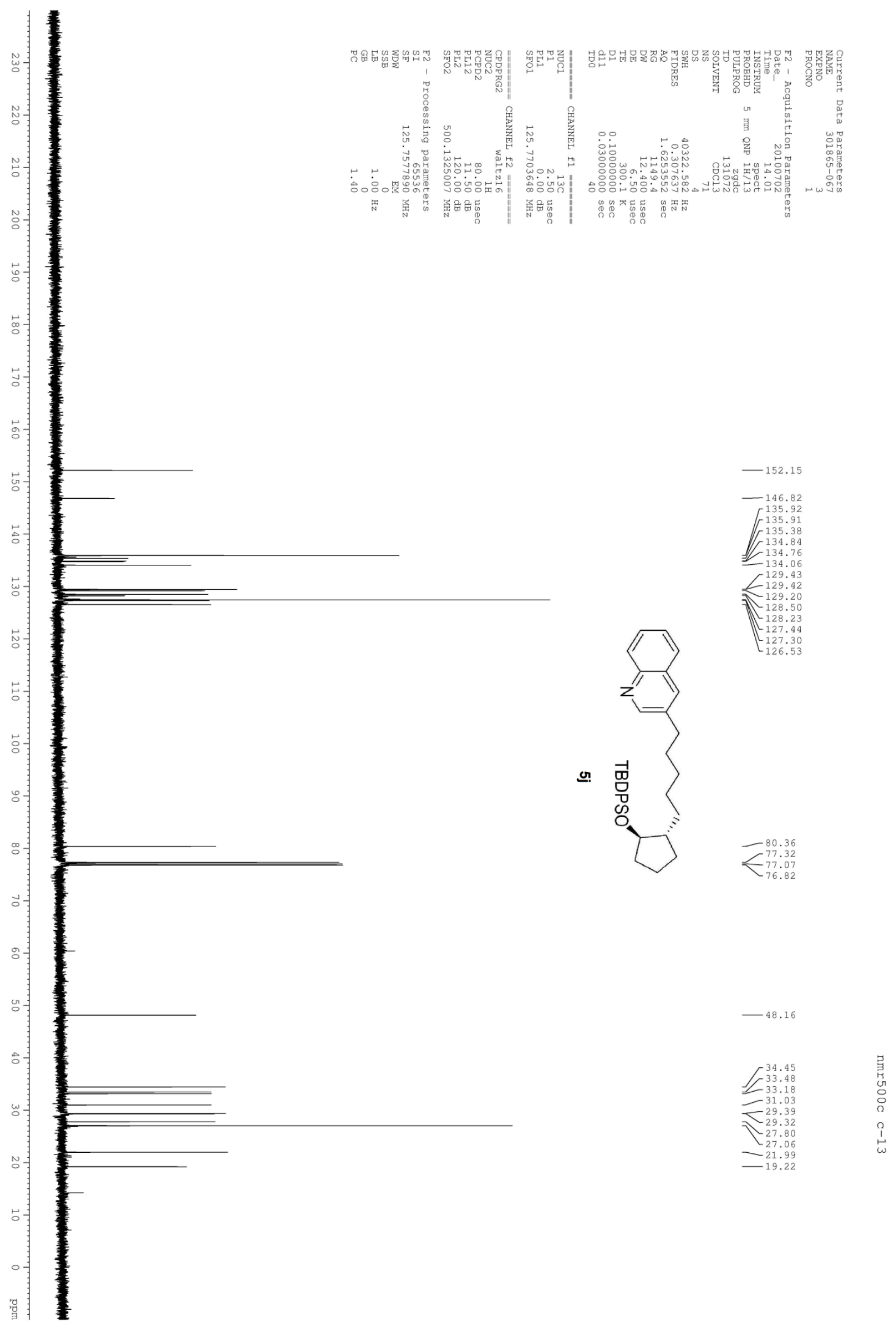




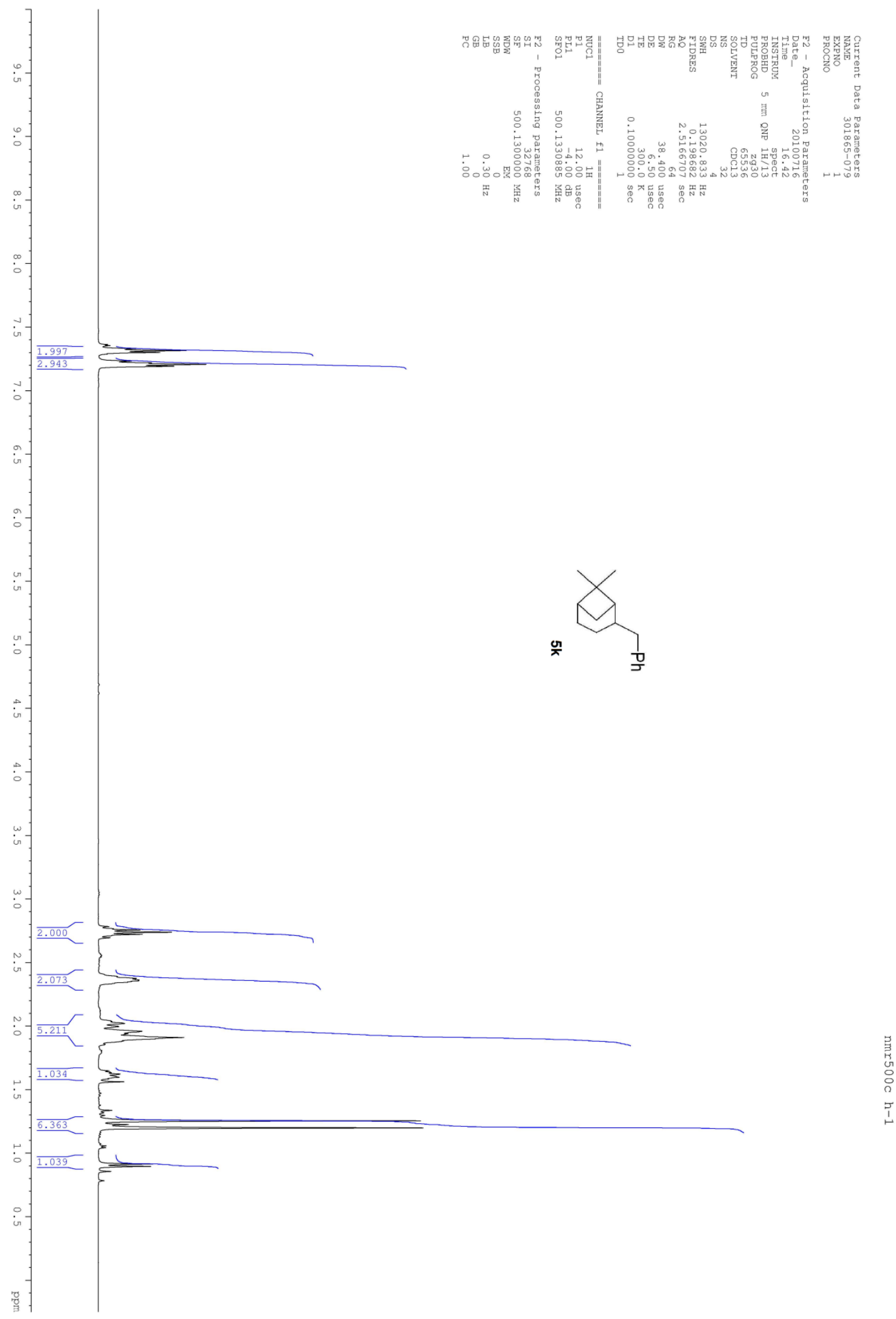



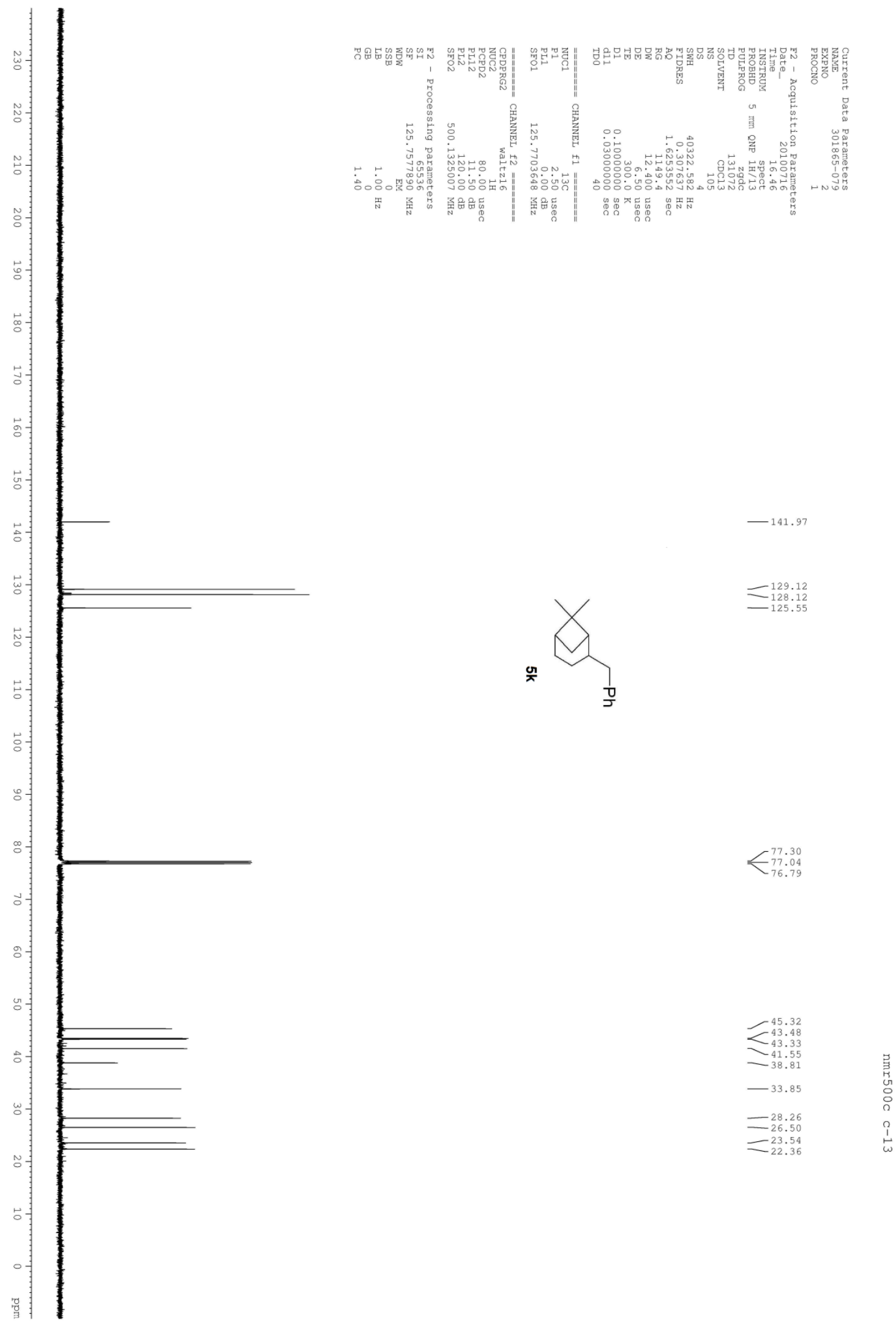


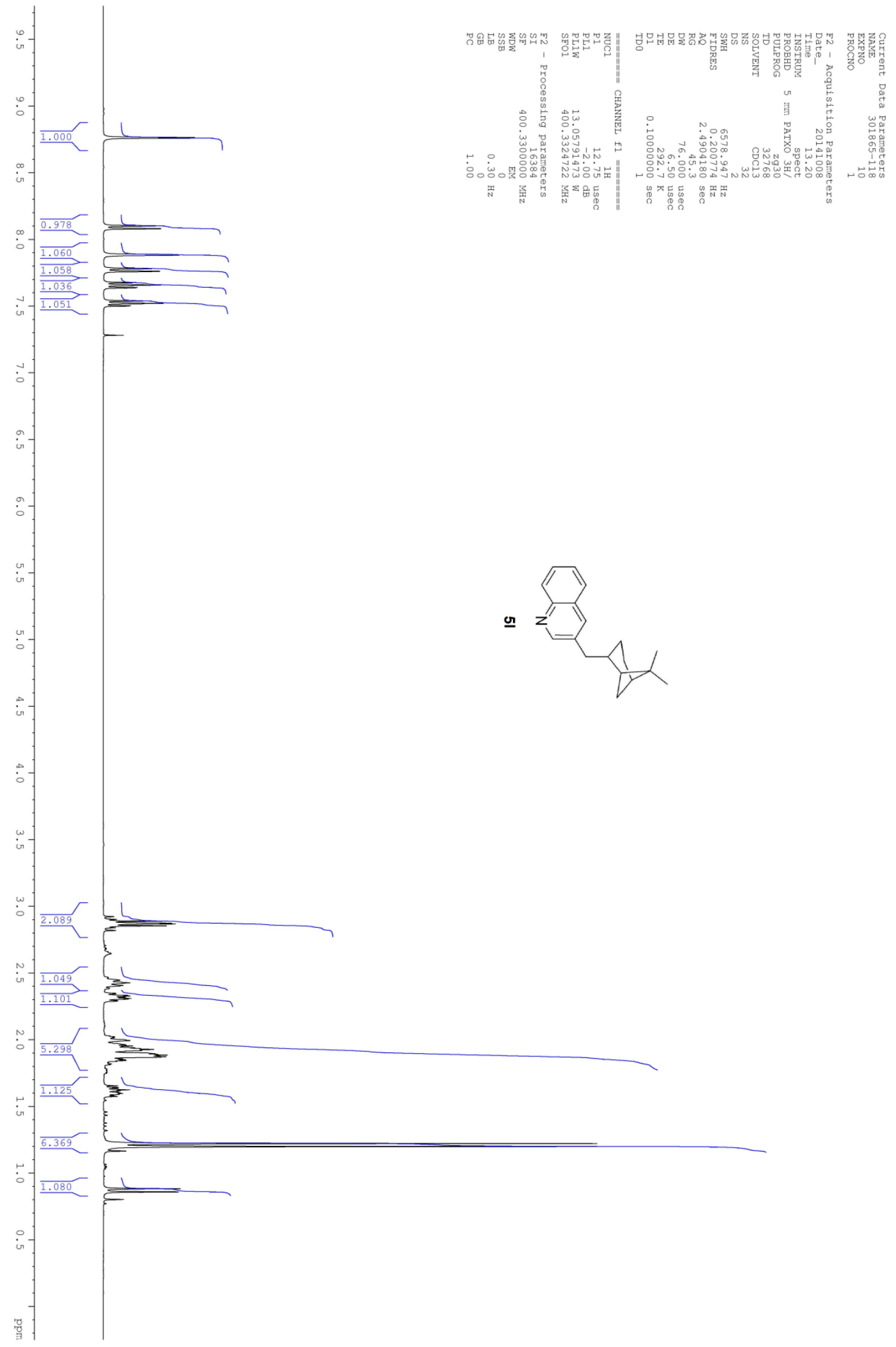



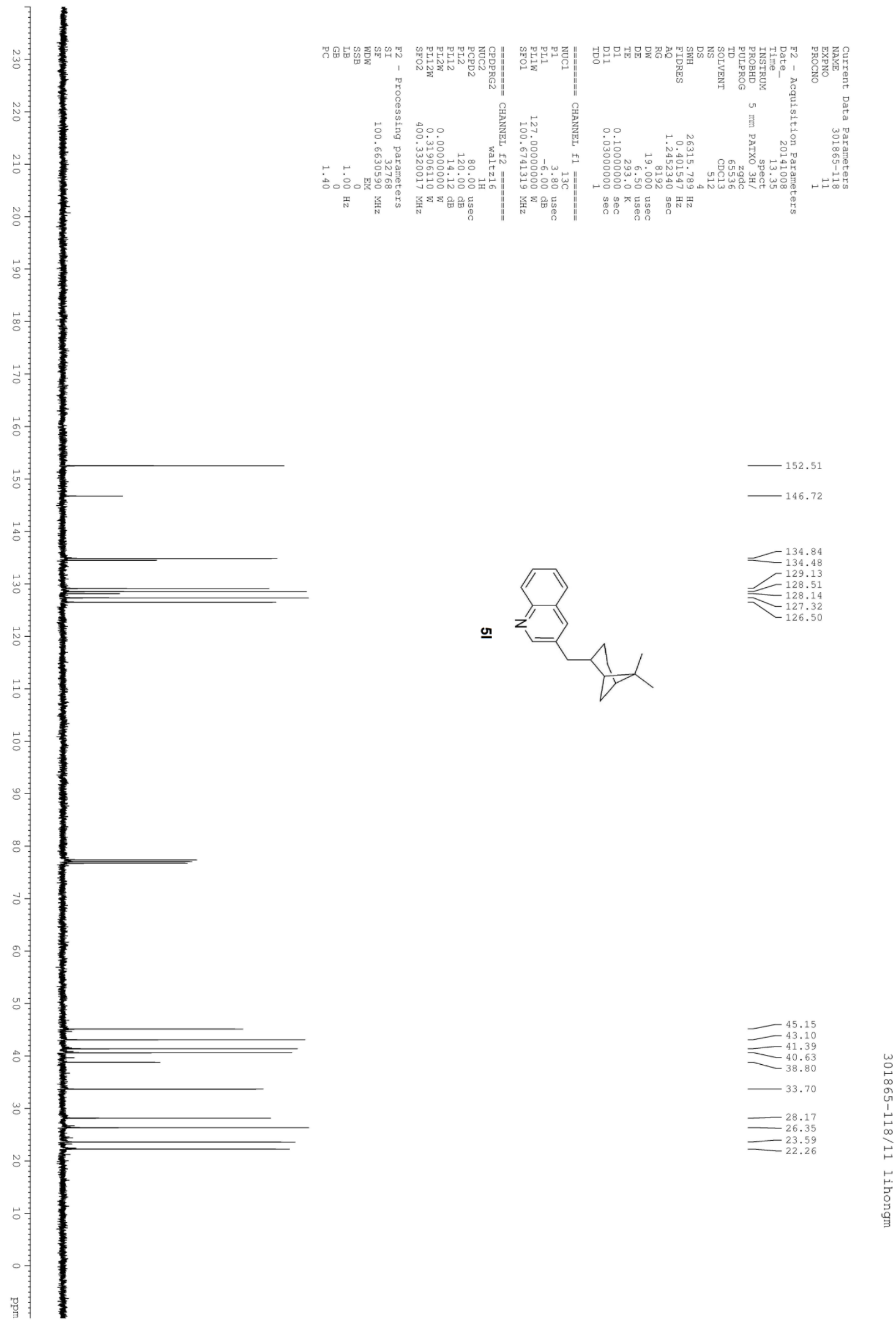


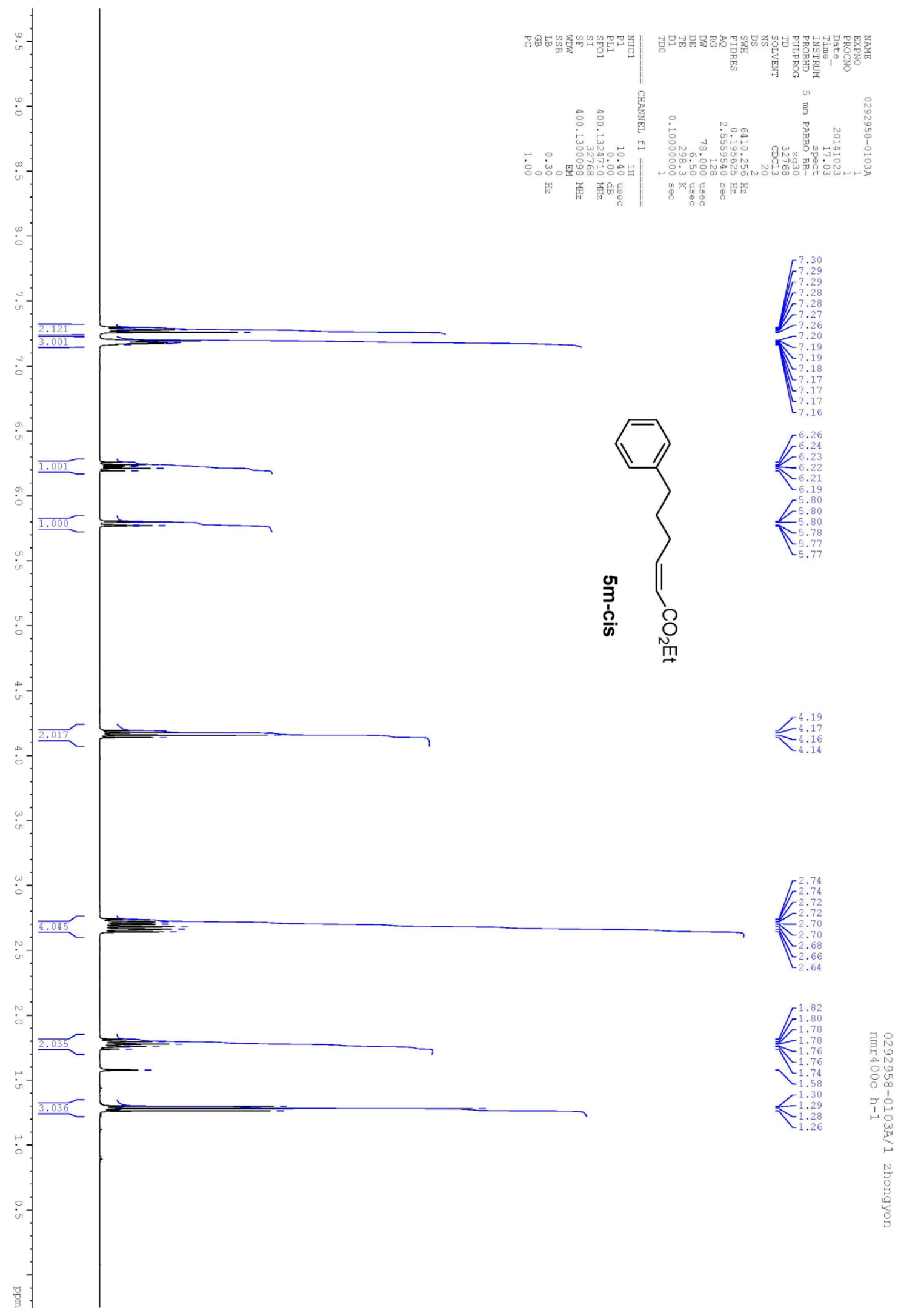



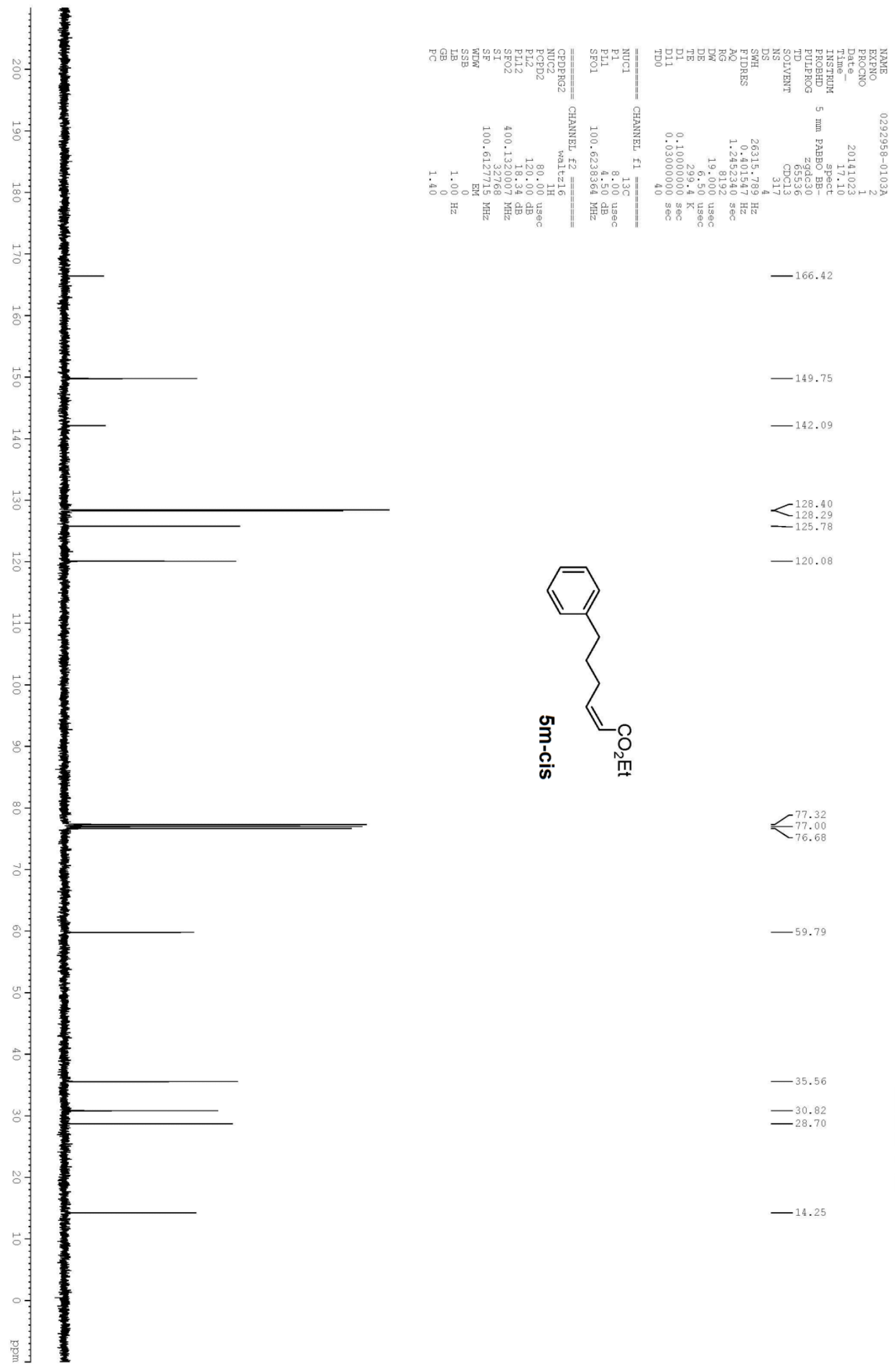

$-166.42$

$-149.75$

$-142.09$
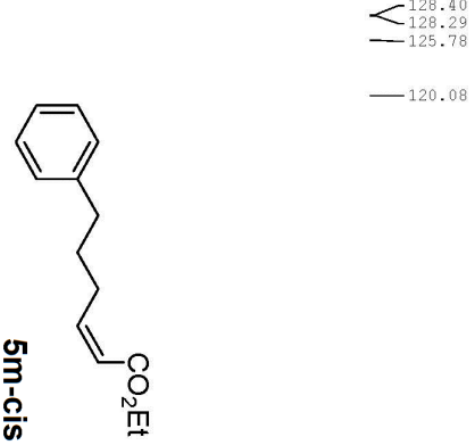

$-120.08$

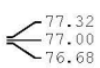

$-59.79$

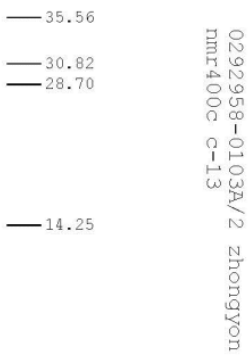




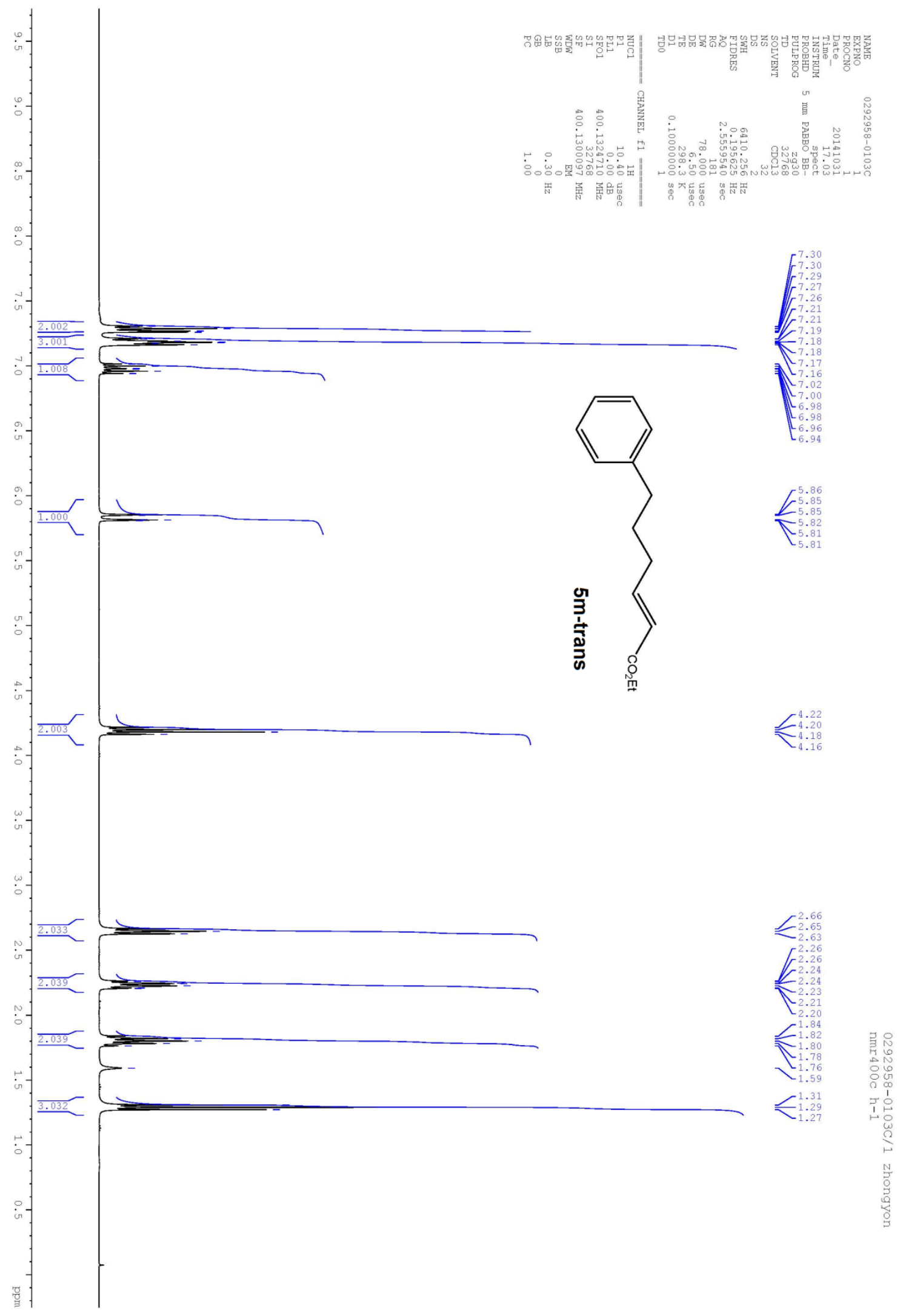



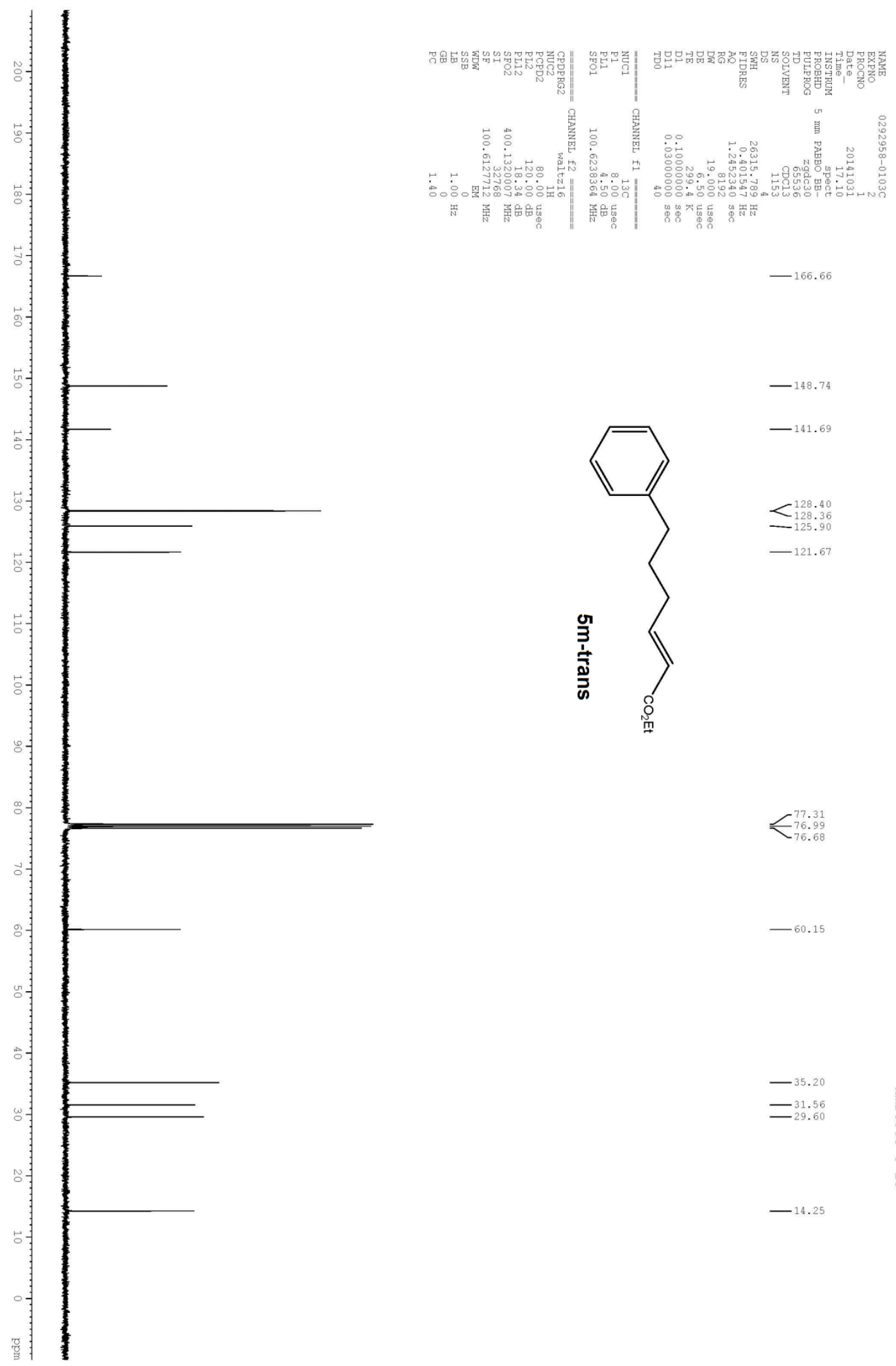

$-166.66$
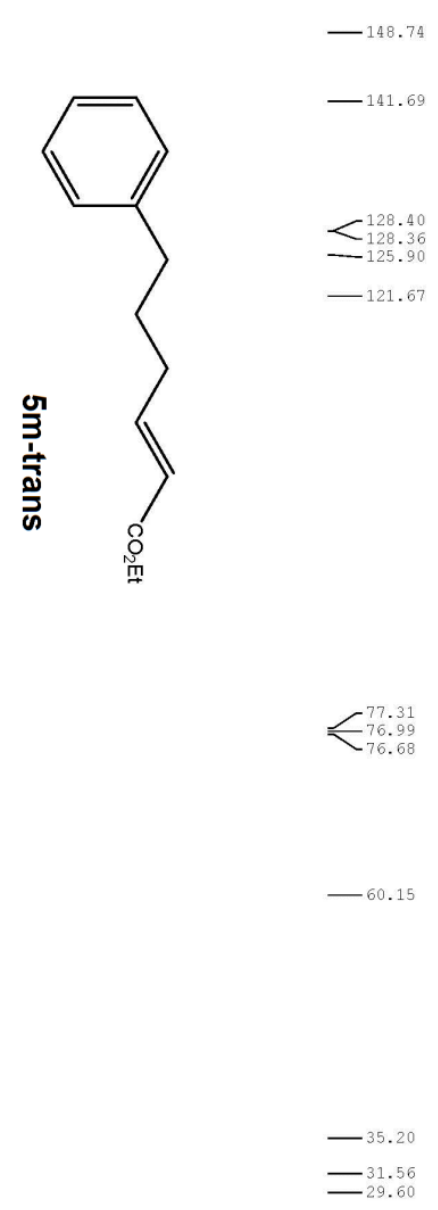

$-14.25$ 


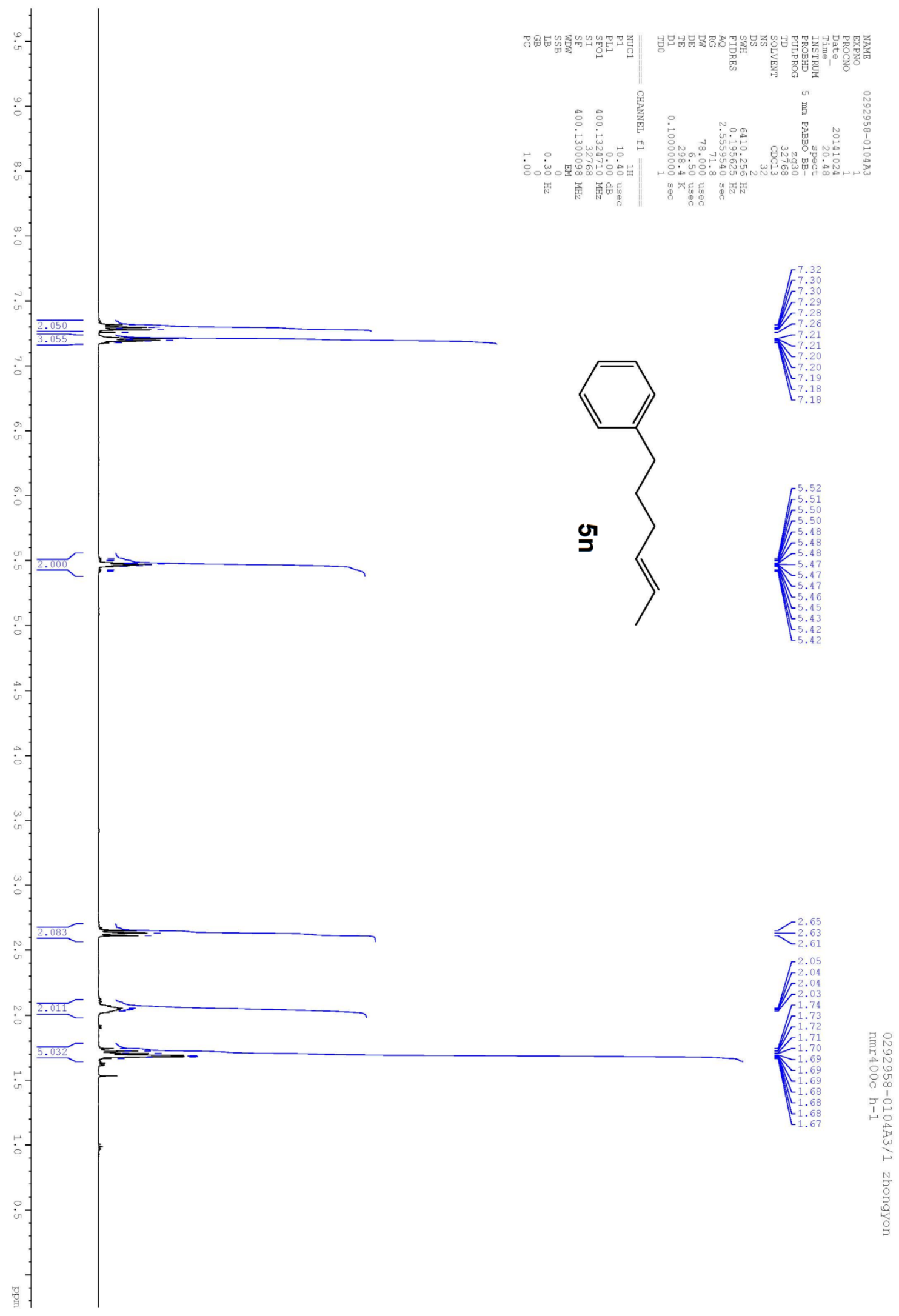



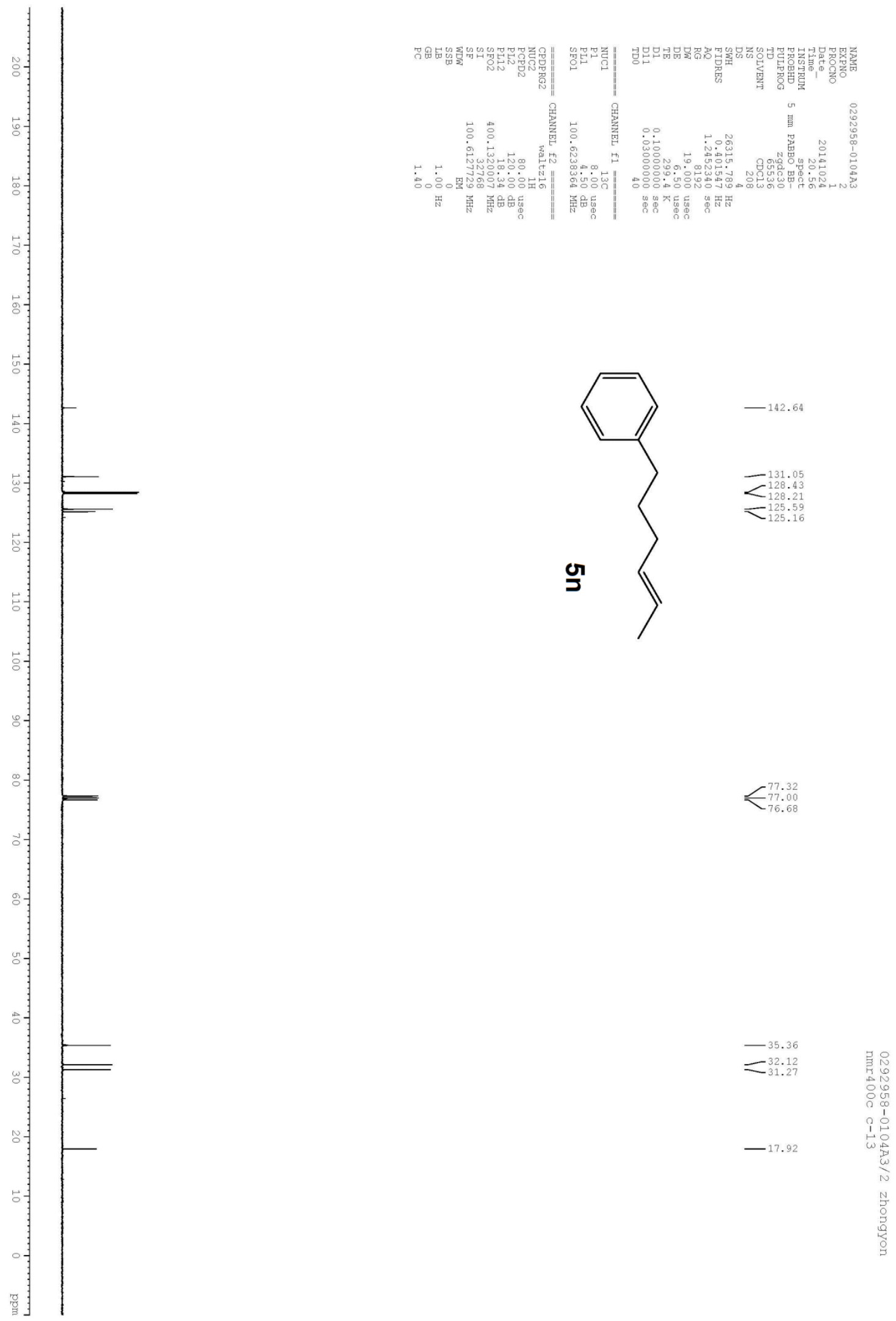


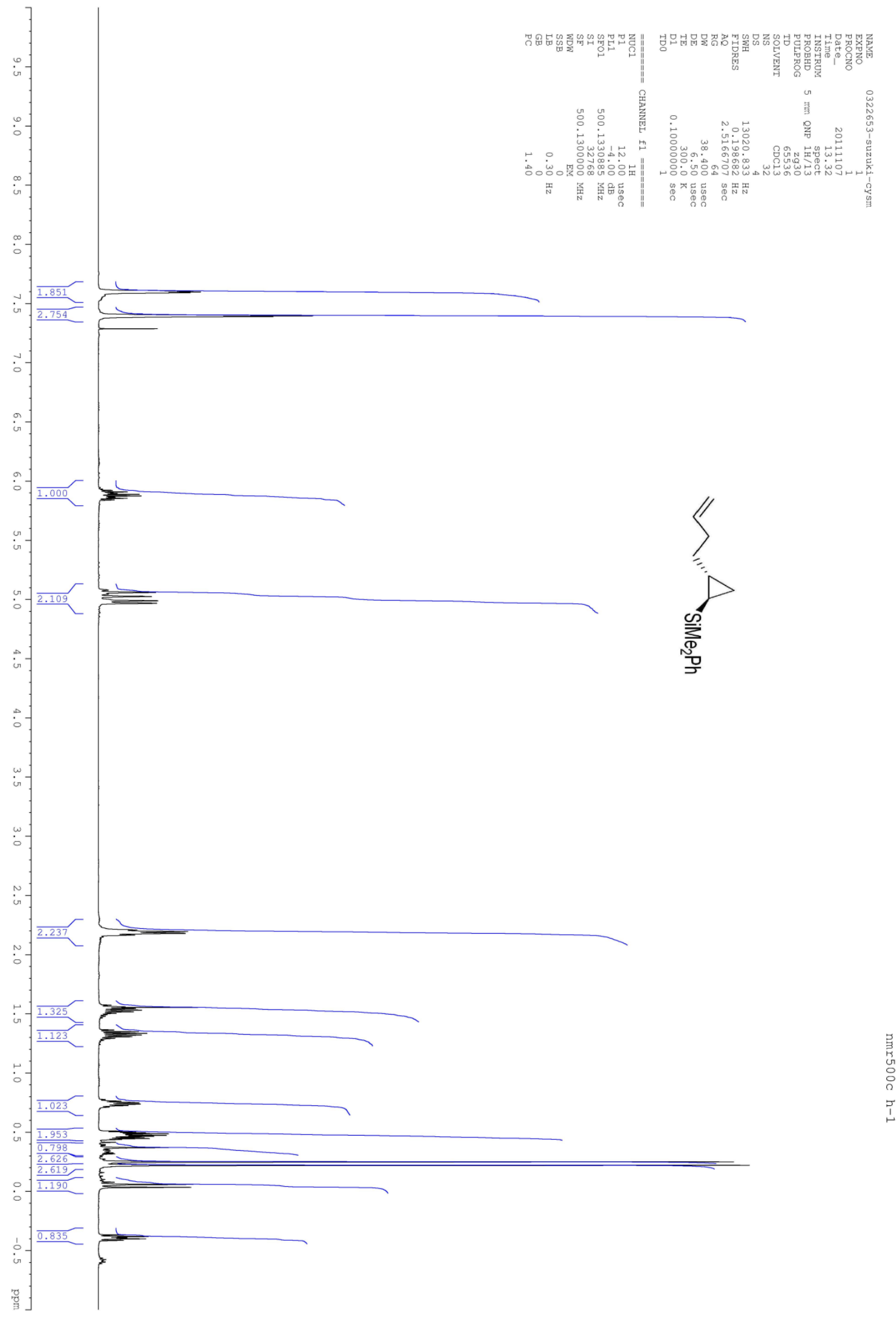



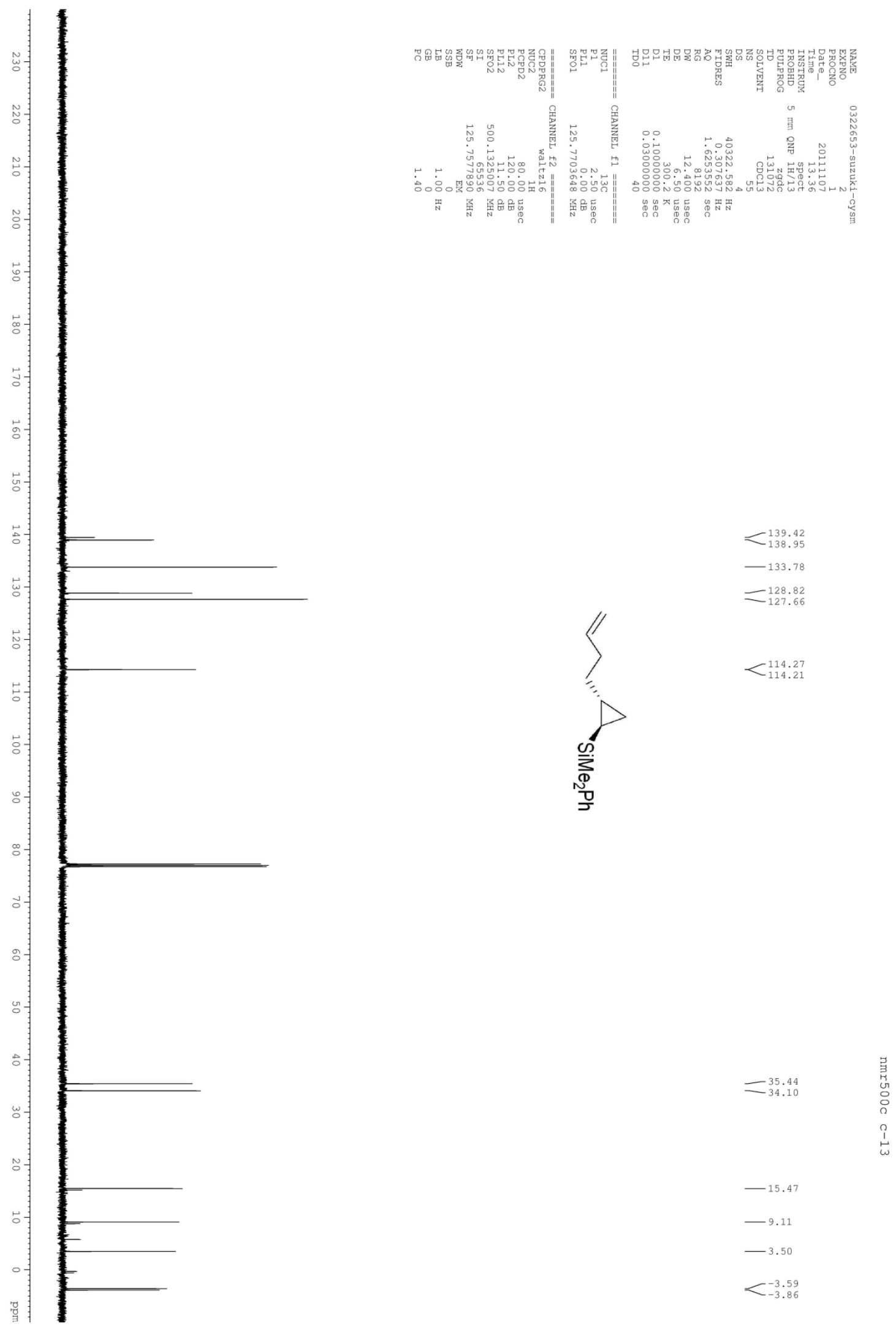

$-15.47$

$-9.11$

$-3.50$

$<_{-3.86}^{-3.59}$ 


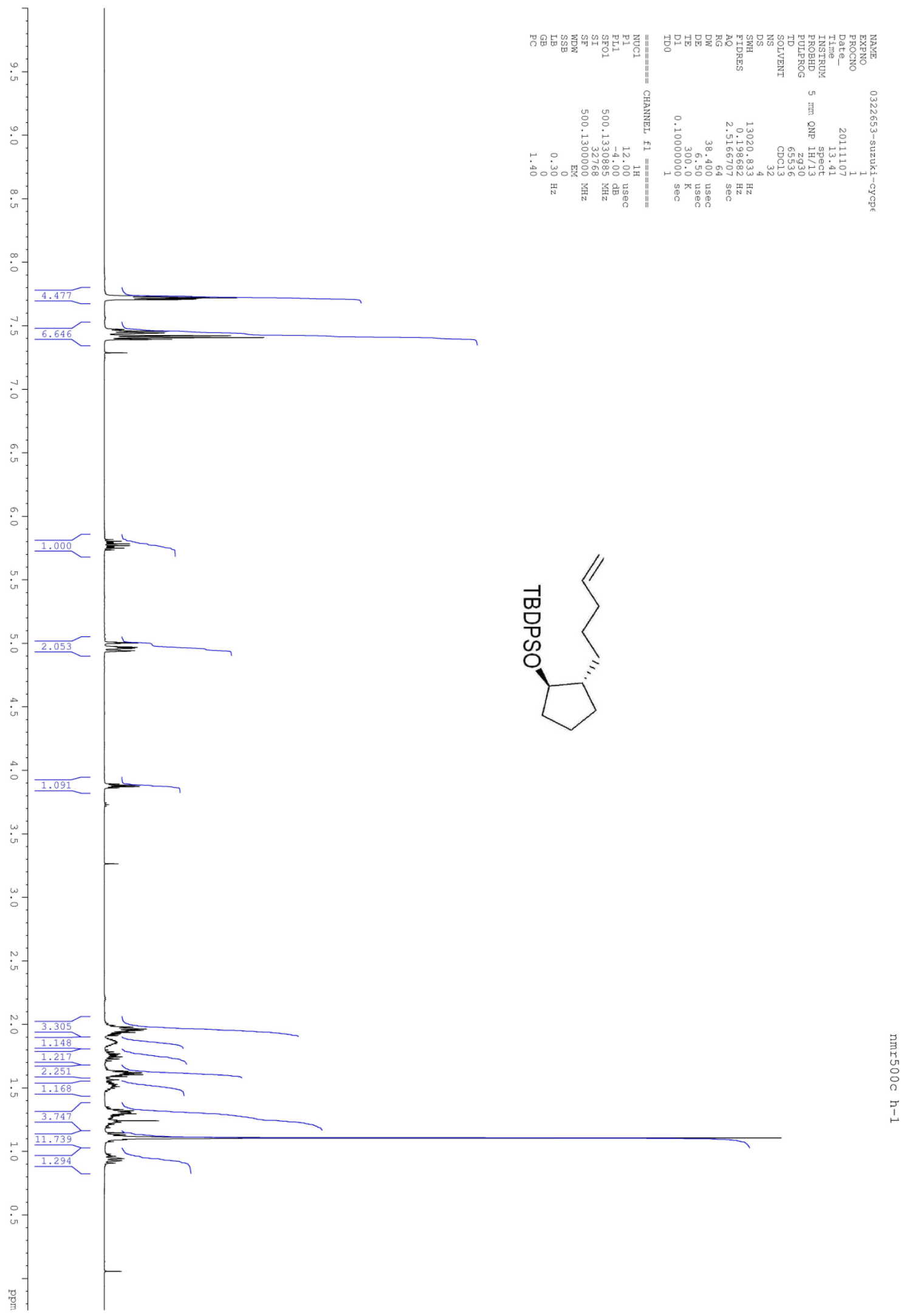




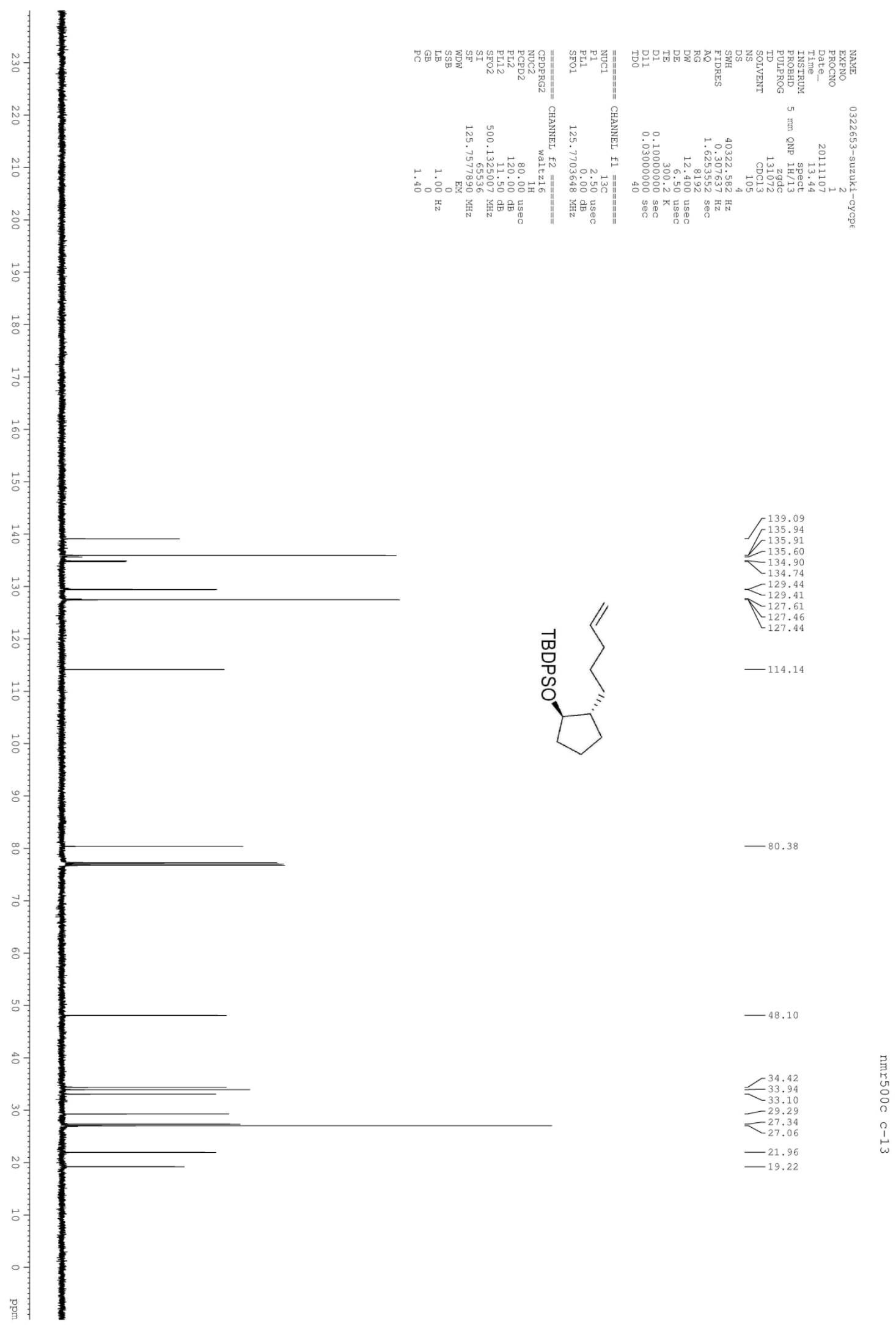


'Matched the commercial available compound.

ii Known compound, see: Oriyama, T.; Yatabe, K.; Sugawara, S.; Machiguchi, Y.; Koga, G. Synlett. 1996, 523-525. 